\title{
Ergodic transforms associated to general averages
}

\author{
by \\ H. Aimar (Santa Fe), A. L. Bernardis (Santa Fe), \\ and F. J. MARtín-Reyes (Málaga)
}

\begin{abstract}
Jones and Rosenblatt started the study of an ergodic transform which is analogous to the martingale transform. In this paper we present a unified treatment of the ergodic transforms associated to positive groups induced by nonsingular flows and to general means which include the usual averages, Cesàro- $\alpha$ averages and Abel means. We prove the boundedness in $L^{p}, 1<p<\infty$, of the maximal ergodic transforms assuming that the semigroup is Cesàro bounded in $L^{p}$. For $p=1$ we find that the maximal ergodic transforms are of weak type $(1,1)$. Convergence results are also proved. We give some general examples of Cesàro bounded semigroups.
\end{abstract}

1. Introduction and main results. Let $(X, \mathcal{F}, \nu)$ be a complete $\sigma$ finite measure space. By a flow $\Gamma=\left\{\tau_{t}: t \in \mathbb{R}\right\}$ we mean a group of measurable transformations $\tau_{t}: X \rightarrow X$ such that $\tau_{0}$ is the identity, $\tau_{t+s}=$ $\tau_{t} \circ \tau_{s}(t, s \in \mathbb{R})$ and the map $(x, t) \mapsto \tau_{t} x$ from $X \times \mathbb{R}$ into $X$ is $\widetilde{\mathcal{F}}-\mathcal{F}$ measurable, where $\widetilde{\mathcal{F}}$ is the completion of the product $\sigma$-algebra $\mathcal{F} \otimes \mathcal{L}$ where $\mathcal{L}$ is the Lebesgue $\sigma$-algebra, and the completion is taken with respect to the product measure of $\nu$ on $\mathcal{F}$ and the Lebesgue measure $m$. The flow is said to be measure preserving if $\nu\left(\tau_{t} E\right)=\nu(E)$ for all $t \in \mathbb{R}$ and all $E \in \mathcal{F}$ (we also say that the flow preserves the measure $\nu$ ). The flow is said to be nonsingular if $\nu\left(\tau_{t} E\right)=0$ for all $t \in \mathbb{R}$ and all $E \in \mathcal{F}$ with $\nu(E)=0$. It is clear that measure preserving implies nonsingular. In this paper we are mainly interested in nonsingular flows which are not necessarily measure preserving.

From now on we fix a nonsingular flow $\Gamma=\left\{\tau_{t}: t \in \mathbb{R}\right\}$. For each $t \in \mathbb{R}$ we consider the measures $\nu_{t}$ defined by $\nu_{t}(E)=\nu\left(\tau_{t}(E)\right)$. These measures have the same sets of measure zero since the flow is nonsingular. If $\overline{J_{t}}$ is the Radon-Nikodym derivative of $\nu_{t}$ with respect to $\nu$ then $\int_{X} f(x) d \nu(x)=$ $\int_{X} f\left(\tau_{t} x\right) \overline{J_{t}}(x) d \nu(x)$ for all nonnegative measurable functions $f$ and for all

2010 Mathematics Subject Classification: 47A35, 37A40, 42B25.

Key words and phrases: ergodic transforms, averages, Cesàro averages, Abel means, weights, Cesàro bounded semigroups, weights, weighted inequalities. 
integrable functions $f$. Moreover, $\overline{J_{t+s}}(x)=\overline{J_{s}}\left(\tau_{t} x\right) \overline{J_{t}}(x)$ a.e. $x$. It follows that the operators $S^{t} f(x)=\overline{J_{t}}(x) f\left(\tau_{t} x\right)$ are positive isometries in $L^{1}(\nu)$ and $\lim _{t \rightarrow 0} S^{t}=I$ in the strong operator topology [15, where $I$ is the identity operator. Consequently, by [6, Lemma III.11.16] (see also [23]) there exists a function $(x, t) \mapsto J_{t}(x)$, measurable with respect to the product $\sigma$-algebra, such that, for almost every $t, J_{t}(x)=\overline{J_{t}}(x)$ a.e. $x$. Consequently,

$$
\int_{X} f(x) d \nu(x)=\int_{X} f\left(\tau_{t} x\right) J_{t}(x) d \nu(x)
$$

for almost every $t$. Furthermore, $J_{t+s}(x)=J_{s}\left(\tau_{t} x\right) J_{t}(x)$ a.e. $(x, s, t) \in X \times$ $\mathbb{R} \times \mathbb{R}$, where in $X \times \mathbb{R} \times \mathbb{R}$ we consider the completion of the product measure.

In this paper we are interested in a class of groups $\mathcal{G}=\left\{T^{t}: t \in \mathbb{R}\right\}$ of positive operators which contains the groups $S^{t}$ considered previously. We introduce this class in the next definition.

Definition 1.1. Let $(X, \mathcal{F}, \nu)$ be a complete $\sigma$-finite measure space. Let $\Gamma=\left\{\tau_{t}: t \in \mathbb{R}\right\}$ be a nonsingular flow on $X$. Let $g(x, t)=g_{t}(x)$ be a positive function defined on $X \times \mathbb{R}$ that is $\mathcal{F} \otimes \mathcal{L}$-measurable and such that $g_{t+s}(x)=g_{s}\left(\tau_{t} x\right) g_{t}(x)$ a.e. $(x, s, t) \in X \times \mathbb{R} \times \mathbb{R}$. A group $\mathcal{G}=\left\{T^{t}: t \in \mathbb{R}\right\}$ of positive operators induced by $\Gamma$ and $g$ is a family of linear operators acting on measurable functions such that $T^{t+s} f=T^{t}\left(T^{s} f\right), T^{0} f=f$, and, for almost every $t$,

$$
T^{t} f(x)=g_{t}(x) f\left(\tau_{t} x\right) \quad \text { a.e. } x .
$$

The semigroup $\left\{T^{t}: t>0\right\}$ will be denoted by $\mathcal{G}_{+}$.

Throughout the paper we work only with this kind of groups. For such a group, it follows from (1.1) that if $0<p<\infty$ and $H_{t}(x)=\left(g_{t}(x)\right)^{-p} J_{t}(x)$ then for almost every $t$,

$$
\int_{X}|f(x)|^{p} d \nu(x)=\int_{X}\left|T^{t} f(x)\right|^{p} H_{t}(x) d \nu(x) .
$$

One of the classical problems in Ergodic Theory is to study the convergence of the averages

$$
\mathcal{A}_{\varepsilon}^{+} f(x)=\frac{1}{\varepsilon} \int_{0}^{\varepsilon} T^{t} f(x) d t
$$

as $\varepsilon \rightarrow 0^{+}$and as $\varepsilon \rightarrow \infty$. (In principle these averages are well defined for $f \geq 0$.) There are other kinds of averages like the Cesàro- $\alpha$ averages

$$
\mathcal{C}_{\varepsilon}^{+} f(x)=\frac{1}{\varepsilon^{1+\alpha}} \int_{0}^{\varepsilon}(\varepsilon-t)^{\alpha} T^{t} f(x) d t, \quad \alpha \geq 0,
$$


or Abel means

$$
\mathcal{R}_{\varepsilon}^{+} f(x)=\frac{1}{\varepsilon} \int_{0}^{\infty} e^{-t / \varepsilon} T^{t} f(x) d t,
$$

which have also been studied in this ergodic setting ([2], [5, 8], [9], [17, [22, [23]). All these averages are particular cases of the "convolution" averages defined by

$$
\mathcal{A}_{\varepsilon, \varphi}^{+} f(x)=\int_{0}^{\infty} \varphi_{\varepsilon}(t) T^{t} f(x) d t
$$

where $\varphi_{\varepsilon}(t)=(1 / \varepsilon) \varphi(t / \varepsilon)$ and $\varphi$ is a nonnegative integrable decreasing function defined on $(0, \infty)$ (the trivial case $\varphi(t)=0$ will not be considered). If $\varphi$ is the characteristic function $\chi_{(0,1)}$ of the interval $(0,1)$ then the $\varphi$-averages $\mathcal{A}_{\varepsilon, \varphi}^{+} f$ are the usual ergodic averages; in this case, as we have already done, we simply write $\mathcal{A}_{\varepsilon}^{+} f$ (this convention will also be used for other operators).

In order to prove the almost everywhere convergence of the averages $\mathcal{A}_{\varepsilon, \varphi}^{+} f$, the standard approach is to consider the maximal operator

$$
\mathcal{M}_{\varphi}^{+} f(x)=\sup _{\varepsilon>0}\left|\mathcal{A}_{\varepsilon, \varphi}^{+} f(x)\right|
$$

and, for $f \in L^{p}(\nu), 1<p<\infty$, to prove a dominated ergodic estimate, i.e., $\int_{X}\left|\mathcal{M}_{\varphi}^{+} f\right|^{p} d \nu \leq C \int_{X}|f|^{p} d \nu$ with a constant $C$ independent of $f$. It is clear that for such an inequality to hold the averages $\mathcal{A}_{\varepsilon, \varphi}^{+}$must be uniformly bounded operators in $L^{p}(\nu)$. This remark gives rise to the next definition.

Definition 1.2. Let $\mathcal{G}$ be a group as in Definition 1.1. Let $\varphi$ be a nonnegative integrable decreasing function on $(0, \infty)$. Let $1 \leq p<\infty$. We say that the semigroup $\mathcal{G}_{+}=\left\{T^{t}: t>0\right\}$ is $\varphi$-bounded in $L^{p}(\nu)$ if there exists $C>0$ such that for all nonnegative functions $f \in L^{p}(\nu)$,

$$
\sup _{\varepsilon>0} \int_{X}\left|\mathcal{A}_{\varepsilon, \varphi}^{+} f\right|^{p} d \nu \leq C \int_{X}|f|^{p} d \nu
$$

If $\varphi=\chi_{(0,1)}$ then we say that the semigroup $\mathcal{G}_{+}$is Cesàro bounded in $L^{p}(\nu)$.

Observe that if the semigroup $\mathcal{G}_{+}$is $\varphi$-bounded in $L^{p}(\nu)$ and $f \in L^{p}(\nu)$ then the averages $\mathcal{A}_{\varepsilon, \varphi}^{+} f$ are well defined and (1.4) holds for all $f \in L^{p}(\nu)$. Obviously, the semigroup $\mathcal{G}_{+}$is $\varphi$-bounded in $L^{p}(\nu)$ if $g_{t}(x)=1$ and the flow is measure preserving.

In what follows we look for a relation between $\varphi$-bounded semigroups and Cesàro bounded semigroups (this is probably known, for instance see [9] for Abel means).

Proposition 1.3. Let $\mathcal{G}$ be a group as in Definition 1.1. Let $\varphi$ be a nonnegative integrable decreasing function on $(0, \infty)$ with $\int_{0}^{\infty} \varphi>0$ and let $1 \leq p<\infty$. The semigroup $\mathcal{G}_{+}$is Cesàro bounded in $L^{p}(\nu)$ if and only if 
the semigroup $\mathcal{G}_{+}$is $\varphi$-bounded in $L^{p}(\nu)$. Furthermore, there exists $C>0$ such that $C \mathcal{M}^{+} f(x) \leq \mathcal{M}_{\varphi}^{+} f(x) \leq\left(\int \varphi\right) \mathcal{M}^{+} f(x)$ for all measurable $f$, where $\mathcal{M}^{+} f(x)=\sup _{\varepsilon>0}\left|\mathcal{A}_{\varepsilon}^{+} f(x)\right|$.

Proof. It suffices to consider $f \geq 0$. Notice that if $\varphi(u)>0$ for some $u>0$ then, since $\varphi$ is decreasing, $\mathcal{A}_{\varepsilon, \varphi}^{+} f(x) \geq u \varphi(u) \mathcal{A}_{\varepsilon u}^{+} f(x)$. From this inequality, we see that $\varphi$-bounded implies Cesàro bounded and

$$
\mathcal{M}^{+} f(x) \leq \frac{1}{u \varphi(u)} \mathcal{M}_{\varphi}^{+} f(x) .
$$

On the other hand, if $\varphi$ is a simple function, $\varphi=\sum_{i=1}^{n} c_{i} \chi_{\left(0, b_{i}\right)}, c_{i} \geq 0$, we get

$$
\mathcal{A}_{\varepsilon, \varphi}^{+} f(x)=\sum_{i=1}^{n} c_{i} b_{i} \mathcal{A}_{\varepsilon b_{i}}^{+} f(x) \leq\left(\sum_{i=1}^{n} c_{i} b_{i}\right) \mathcal{M}^{+} f(x)=\left(\int \varphi\right) \mathcal{M}^{+} f(x) .
$$

Then $\mathcal{M}_{\varphi}^{+} f(x) \leq\left(\int \varphi\right) \mathcal{M}^{+} f(x)$ and

$$
\int_{X}\left|\mathcal{A}_{\varepsilon, \varphi}^{+} f\right|^{p} d \nu \leq\left(\int \varphi\right)^{p} \sup _{\varepsilon>0} \int_{X}\left|\mathcal{A}_{\varepsilon}^{+} f\right|^{p} d \nu
$$

Consequently, Cesàro bounded implies $\varphi$-bounded for $\varphi$ simple. For general $\varphi$, the assertion follows from the above inequalities and the monotone convergence theorem.

Proposition 1.3 allows us to reduce the study of the boundedness of $\mathcal{M}_{\varphi}^{+}$to the usual ergodic maximal operator, i.e., to the case $\varphi=\chi_{(0,1)}$ corresponding to the standard ergodic averages. In the next proposition we show that the almost everywhere convergence of $\mathcal{A}_{\varepsilon, \varphi}^{+} f$ also reduces to the standard case.

Proposition 1.4. Let $\mathcal{G}$ be a group as in Definition 1.1. Let $\varphi$ be a nonnegative integrable decreasing function on $(0, \infty)$ with $\int_{0}^{\infty} \varphi>0$ and let $1 \leq p<\infty$. Assume that for a nonnegative measurable function $f$ the limit $\lim _{\varepsilon \rightarrow \infty} \mathcal{A}_{\varepsilon}^{+} f(x)=\ell$ exists and $\mathcal{M}^{+} f(x)<\infty$ for some $x$. Then the limits $\lim _{\varepsilon \rightarrow \infty} \mathcal{A}_{\varepsilon, \varphi}^{+} f(x)=\left(\int \varphi\right) \ell$ exists. The same statement holds for the limit as $\varepsilon$ goes to zero.

Proof. If $\varphi$ is a simple function then the assertion follows immediately from the first equality in 1.5 . Assume now that $\varphi$ has compact support. It is easy to see that there exists a sequence $\left\{\varphi_{n}\right\}_{n}$ of nonnegative decreasing simple functions such that $\varphi_{n} \uparrow \varphi$ and $\varphi-\varphi_{n} \leq \psi_{n}$, where the functions $\psi_{n}$ are nonnegative, integrable, decreasing and $\lim _{n \rightarrow \infty} \int_{0}^{\infty} \psi_{n}=0$. By Proposition 1.3 we have

$$
\left|\mathcal{A}_{\varepsilon, \varphi}^{+} f(x)-\mathcal{A}_{\varepsilon, \varphi_{n}}^{+} f(x)\right|=\mathcal{A}_{\varepsilon, \varphi-\varphi_{n}}^{+} f(x) \leq \mathcal{A}_{\varepsilon, \psi_{n}}^{+} f(x) \leq\left(\int \psi_{n}\right) \mathcal{M}^{+} f(x) .
$$


The result now follows immediately since $\mathcal{M}^{+} f(x)$ is finite, $\lim _{n \rightarrow \infty} \int \psi_{n}=0$ and $\lim _{\varepsilon \rightarrow \infty} \mathcal{A}_{\varepsilon, \varphi_{n}}^{+} f(x)=\left(\int \varphi_{n}\right) \ell$.

Take now a general $\varphi$. Let $L>0$ and $\varphi_{L}=\varphi \chi_{(0, L]}$. Let $\psi_{L}=\varphi(L) \chi_{(0, L]}$ $+\varphi \chi_{(L, \infty)}$. Then

$$
\begin{aligned}
\left|\mathcal{A}_{\varepsilon, \varphi}^{+} f(x)-\left(\int \varphi\right) \ell\right| & \leq\left|\mathcal{A}_{\varepsilon, \varphi_{L}}^{+} f(x)-\left(\int \varphi\right) \ell\right|+\left|\mathcal{A}_{\varepsilon, \psi_{L}}^{+} f(x)\right| \\
& \leq\left|\mathcal{A}_{\varepsilon, \varphi_{L}}^{+} f(x)-\left(\int \varphi\right) \ell\right|+\left(\int \psi_{L}\right) \mathcal{M}^{+} f(x) .
\end{aligned}
$$

Since $\lim _{L \rightarrow \infty} \int \psi_{L}=0$ and $\mathcal{M}^{+} f(x)<\infty$, and $\varphi_{L}$ has compact support, the general case follows from what we have already proved.

Now we are going to give some nontrivial examples of Cesàro bounded semigroups. Some other examples and related questions are studied in $\S 6$.

EXAMPLE 1.5. Let $1 \leq p<\infty$ and $T^{t} f(x)=\left(\overline{J_{t}}(x)\right)^{1 / p} f\left(\tau_{t} x\right)$, where $\overline{J_{t}}$ is the Radon-Nikodym derivative considered at the beginning of the introduction. It is clear that each $T^{t}$ is an isometry on $L^{p}(\nu)$, and consequently $\mathcal{G}_{+}$is Cesàro bounded in $L^{p}(\nu)$. Observe that $\mathcal{G}_{-}=\left\{T^{t}: t<0\right\}$ is also Cesàro bounded in $L^{p}(\nu)$.

EXAMPLE 1.6. Let $X=[0,1)$ with the Lebesgue $\sigma$-algebra. Let $d \nu=$ $w(x) d x$, where $w(x)=x^{\beta}$ and $-1<\beta<0$. Consider the flow $\tau_{t}(x)=x+t$ $(\bmod 1)$, that is, $\tau_{t}(x)=x+t-[x+t]$, where $[x+t]$ stands for the integer part of $x+t$. Set $T^{t} f(x)=f\left(\tau_{t} x\right)$. The semigroup $\mathcal{G}_{+}$is Cesàro bounded in $L^{1}(\nu)$ (and therefore bounded in $L^{p}(\nu), 1<p<\infty$ ) if and only if there exists $C>0$ such that for all $\varepsilon>0$,

$$
\frac{1}{\varepsilon} \int_{0}^{\varepsilon} w\left(\tau_{-t} x\right) d t \leq C w(x) \quad \text { a.e. } x .
$$

This holds since $|x|^{\beta}$ satisfies the Muckenhoupt $A_{1}$ condition on the real line (see [10], 7] or [12]), i.e., there exists $C>0$ such that $(b-a)^{-1} \int_{a}^{b}|x|^{\beta} d x \leq$ $C \inf _{x \in(a, b)}|x|^{\beta}$ for all intervals $(a, b)$. However, the semigroup $\mathcal{G}_{-}=\left\{T^{t}\right.$ : $t<0\}$ is not Cesàro bounded in $L^{1}(\nu)$. To prove this we observe that $\mathcal{G}_{-}$ is Cesàro bounded in $L^{1}(\nu)$ if and only if there exists $C>0$ such that, for all $\varepsilon>0, \varepsilon^{-1} \int_{0}^{\varepsilon} w\left(\tau_{t} x\right) d t \leq C w(x)$ a.e. $x$. By the continuity of $w$, this inequality would hold for all $x \neq 0$. If we take $\varepsilon>0$ and $x=1-\varepsilon$, we have

$$
\frac{1}{2 \varepsilon} \int_{0}^{2 \varepsilon} w\left(\tau_{t} x\right) d t \geq \frac{1}{2 \varepsilon} \int_{0}^{\varepsilon} y^{\beta} d y=\frac{1}{2(\beta+1)} \varepsilon^{\beta} .
$$

Since $w(x)=w(1-\varepsilon)<2$ for $\varepsilon$ small, we see that

$$
\frac{\frac{1}{2 \varepsilon} \int_{0}^{2 \varepsilon} w\left(\tau_{t}(1-\varepsilon)\right) d t}{w(1-\varepsilon)} \geq \frac{1}{4(\beta+1)} \varepsilon^{\beta}
$$


is as large as we wish if we take $\varepsilon$ small enough. Therefore, $\mathcal{G}_{-}$is not Cesàro bounded in $L^{1}(\nu)$.

Clearly, the measure $\nu$ is equivalent to the Lebesgue measure (the invariant measure for the flow $\Gamma$ ) in the sense that they have the same sets of measure zero. However $\nu$ is not comparable to the Lebesgue measure, that is, there is no constant $K>0$ such that $(1 / K)|E| \leq \nu(E) \leq K|E|$ for all measurable sets $E$, where $|E|$ is the Lebesgue measure of $E$ : if $E=(0, b) \subset(0,1)$ we have $\nu(E) /|E|=b^{\beta} /(\beta+1)$ and $b^{\beta}$ is as large as we wish if we take $b$ small enough.

One may ask whether or not the flow could have the property that for some constant $K>0$,

$$
(1 / K) \nu(E) \leq \nu\left(\tau_{t}(E)\right) \leq K \nu(E)
$$

for all measurable sets $E$ and all $t$. Then there should be a $\sigma$-finite measure $\mu$ equivalent to $\nu$ for which the flow is measure preserving and such that $\mu$ is comparable to $\nu$ (consequently, everything would be reduced to the measure preserving case). Our present example shows that the measure $\mu$ would be the Lebesgue measure and we have already shown that it is not comparable to $\nu$. However, we are going to give a direct proof showing that 1.6 does not hold in our example although the semigroup is Cesàro bounded in $L^{1}(\nu)$. In fact, for $0<\varepsilon<1$ and $I_{\varepsilon}=(1-\varepsilon, 1)$, we have $\tau_{\varepsilon}\left(I_{\varepsilon}\right)=(0, \varepsilon)$. Therefore, $\nu\left(\tau_{\varepsilon}\left(I_{\varepsilon}\right)\right)=\varepsilon^{\beta+1} /(\beta+1)$. Since $\nu\left(I_{\varepsilon}\right) \leq 2^{-\beta} \varepsilon$, we have

$$
\frac{\nu\left(\tau_{\varepsilon}\left(I_{\varepsilon}\right)\right)}{\nu\left(I_{\varepsilon}\right)} \geq \frac{2^{\beta} \varepsilon^{\beta}}{\beta+1}
$$

which is as large as we wish if we take $\varepsilon$ small enough.

In what follows we state our results about the boundedness of $\mathcal{M}_{\varphi}^{+}$and the convergence of the averages $\mathcal{A}_{\varepsilon, \varphi}^{+} f$ under the main assumption that the semigroup $\mathcal{G}_{+}$is Cesàro bounded in $L^{p}(\nu)$.

Theorem 1.7. Let $(X, \mathcal{F}, \nu), \Gamma,\left\{g_{t}: t \in \mathbb{R}\right\}$ and $\mathcal{G}=\left\{T^{t}: t \in \mathbb{R}\right\}$ be as in Definition 1.1. Let $\varphi$ be a nonnegative integrable decreasing function on $(0, \infty)$ and let $1 \leq p<\infty$. Assume that the semigroup $\mathcal{G}_{+}$is Cesàro bounded in $L^{p}(\nu)$.

(a) If $1<p<\infty$ then:

(i) There exists $C>0$ such that for all $f \in L^{p}(\nu)$,

$$
\int_{X}\left|\mathcal{M}_{\varphi}^{+} f\right|^{p} d \nu \leq C \int_{X}|f|^{p} d \nu
$$

(ii) For all $f \in L^{p}(\nu), \lim _{\varepsilon \rightarrow 0^{+}} \mathcal{A}_{\varepsilon, \varphi} f=\left(\int \varphi\right) f$ a.e. and in $L^{p}(\nu)$.

(iii) For all $f \in L^{p}(\nu)$, the averages $\mathcal{A}_{\varepsilon, \varphi}^{+} f$ converge a.e. and in $L^{p}(\nu)$ as $\varepsilon \rightarrow \infty$. 
(b) If $p=1$ and $g_{t}(x)=1$ then:

(i) There exists $C>0$ such that for all $f \in L^{1}(\nu)$ and all $\lambda>0$,

$$
\nu\left(\left\{x \in X:\left|\mathcal{M}_{\varphi}^{+} f(x)\right|>\lambda\right\}\right) \leq \frac{C}{\lambda} \int_{X}|f|^{p} d \nu .
$$

(ii) For all $f \in L^{1}(\nu), \lim _{\varepsilon \rightarrow 0^{+}} \mathcal{A}_{\varepsilon, \varphi}^{+} f=\left(\int \varphi\right) f$ a.e. and in measure.

(iii) For all $f \in L^{1}(\nu)$, the averages $\mathcal{A}_{\varepsilon, \varphi}^{+} f$ converge a.e. and in measure as $\varepsilon \rightarrow \infty$.

REMARK 1.8. The result does not hold if $p=1$ and $g_{t}(x) \neq 1$. To show an example we work on the real line. We follow Example 2.11 in [11]. Take

$$
g(x)=\chi_{(-\infty, 1)}(x)+\frac{1}{x} \chi_{(1, \infty)}(x), \quad T^{t} f(x)=\frac{g(x-t)}{g(x)} f(x-t)
$$

and $d \nu=g(x) d x$. It is easy to see that the semigroup $\mathcal{G}_{+}$is Cesàro bounded in $L^{1}(d \nu)$. Notice that the maximal operator $\mathcal{M}^{+}$associated to the semigroup satisfies

$$
\mathcal{M}^{+} f(x)=\frac{1}{g(x)} M^{-}(f g)(x),
$$

where $M^{-}$is the one-sided Hardy-Littlewood maximal function defined by $M^{-} f(x)=\sup _{\varepsilon>0} \varepsilon^{-1} \int_{x-\varepsilon}^{x}|f(s)| d s$. Therefore, if a weak type $(1,1)$ inequality were satisfied for $\mathcal{M}^{+}$with respect to $\nu$ we would have

$$
\int_{\left\{x:(1 / g(x)) M^{-} f(x)>\lambda\right\}} g(x) d x \leq \frac{C}{\lambda} \int_{\mathbb{R}}|f(x)| d x .
$$

If we take $f=\chi_{(0,1)}$ then $M^{-} f(x)=1 / x$ for $x>1$. Taking $\lambda=1 / 2$, we have $\infty=\int_{(1, \infty)} g(x) d x \leq 2 C \int_{\mathbb{R}}|f(x)| d x=2 C$.

Once we know that the convergence of $\mathcal{A}_{\varepsilon, \varphi}^{+} f$ holds in the almost everywhere sense or in the $L^{p}$-norm, it is reasonable to try to give some information about how the convergence occurs.

Take any sequence $\left\{\varepsilon_{k}\right\}_{k \in \mathbb{Z}}$ with $\varepsilon_{k+1}>\varepsilon_{k}>0$ for all $k, \lim _{k \rightarrow-\infty} \varepsilon_{k}=0$ and $\lim _{k \rightarrow \infty} \varepsilon_{k}=\infty$. Nothing can be said about the rate of convergence of $\lim _{k \rightarrow-\infty} \mathcal{A}_{\varepsilon_{k}, \varphi}^{+} f$. However, for $N \leq 0, \sum_{k=N}^{0}\left(\mathcal{A}_{\varepsilon_{k}, \varphi} f(x)-\mathcal{A}_{\varepsilon_{k-1}, \varphi} f(x)\right)=$ $\mathcal{A}_{\varepsilon_{0}, \varphi} f(x)-\mathcal{A}_{\varepsilon_{N-1}, \varphi} f(x)$. Therefore, the limit

$$
\begin{aligned}
\lim _{N \rightarrow-\infty} \sum_{k=N}^{0}\left(\mathcal{A}_{\varepsilon_{k}, \varphi} f(x)-\mathcal{A}_{\varepsilon_{k-1}, \varphi} f(x)\right) & \\
& =\mathcal{A}_{\varepsilon_{0}, \varphi} f(x)-\lim _{N \rightarrow-\infty} \mathcal{A}_{\varepsilon_{N-1}, \varphi} f(x)
\end{aligned}
$$

exists and is essentially equal to $\lim _{k \rightarrow-\infty} \mathcal{A}_{\varepsilon_{k}, \varphi}^{+} f$. Consequently, if we try to give some information about the convergence of $\mathcal{A}_{\varepsilon_{k}, \varphi}^{+} f$, we can analyze 
the convergence of $\sum_{k=-\infty}^{0}\left(\mathcal{A}_{\varepsilon_{k}, \varphi} f(x)-\mathcal{A}_{\varepsilon_{k-1}, \varphi} f(x)\right)$. We can try to prove the absolute convergence, the unconditional convergence or the existence in $L^{p}(\nu)$ of the square function $\sum_{k=-\infty}^{\infty}\left|\mathcal{A}_{\varepsilon_{k}, \varphi} f-\mathcal{A}_{\varepsilon_{k-1}, \varphi} f\right|^{2}$. If, in particular, we take a lacunary sequence $\left\{\varepsilon_{k}\right\}_{k \in \mathbb{Z}}$, i.e., $\varepsilon_{k}>0$ and $\varepsilon_{k+1} / \varepsilon_{k} \geq \rho>1$ for all $k$, then an example in [1] shows that there exists $f \in L^{\infty}(\nu)$ such that the series does not converge absolutely. More precisely, let $X=[0,1)$ with the Lebesgue measure $\nu$ and, as before, consider the flow $\tau_{t}(x)=$ $x+t(\bmod 1)$. Let $\varphi=\chi_{(0,1)}$. Then there exists $f \in L^{\infty}(\nu)$ such that $\sum_{k=-\infty}^{0}\left|\mathcal{A}_{\varepsilon_{k}, \varphi} f(x)-\mathcal{A}_{\varepsilon_{k-1}, \varphi} f(x)\right|=\infty$ a.e. (The example in [1] is for $\varphi=\chi_{(-1,1)}$, that is, $\mathcal{A}_{\varepsilon} f(x)=(2 \varepsilon)^{-1} \int_{-\varepsilon}^{\varepsilon} f(x+t) d t$, but the example for $\varphi=\chi_{(0,1)}$ follows immediately.) Given the cancellation properties of $\sum_{k=-\infty}^{0}\left(\mathcal{A}_{\varepsilon_{k}, \varphi} f(x)-\mathcal{A}_{\varepsilon_{k-1}, \varphi} f(x)\right)$ and the last result, it is natural to consider the convergence of

$$
\sum_{k=-\infty}^{0} v_{k}\left(\mathcal{A}_{\varepsilon_{k}, \varphi} f(x)-\mathcal{A}_{\varepsilon_{k-1}, \varphi} f(x)\right),
$$

where $v_{k}$ is a bounded sequence of real or complex numbers. Reasoning in the same way for $k \rightarrow \infty$, we arrive at the problem of convergence of the series $\sum_{k=-\infty}^{\infty} v_{k}\left(\mathcal{A}_{\varepsilon_{k}, \varphi} f(x)-\mathcal{A}_{\varepsilon_{k-1}, \varphi} f(x)\right)$, where $v_{k}$ is a bounded sequence of real or complex numbers. We shall only study the convergence and boundedness of the last series for lacunary sequences because the expected results imply unconditional convergence of the series $\sum_{k=-\infty}^{\infty}\left(\mathcal{A}_{\varepsilon_{k}, \varphi} f(x)-\mathcal{A}_{\varepsilon_{k-1}, \varphi} f(x)\right)$, and this fact restricts the classes of sequences for which you can expect positive results (see Remark 1.15 after the statement of the results). We must point out that Jones and Rosenblatt [14] studied this problem for $\varphi=\chi_{(0,1)}$ in the setting of periodic functions on the real line with the flow $\tau_{t}(x)=x+t$ and for discrete averages associated to a measure preserving transformation; the problem for general functions on the real line with the same flow and in the context of weighted spaces was studied in [3].

So far, we have only established our main aim, that is, to study the convergence and boundedness of

$$
\sum_{k=-\infty}^{\infty} v_{k}\left(\mathcal{A}_{\varepsilon_{k}, \varphi} f(x)-\mathcal{A}_{\varepsilon_{k-1}, \varphi} f(x)\right)
$$

in the setting of Cesàro bounded semigroups, where $\varepsilon_{k}$ is a lacunary sequence and $v_{k}$ is a bounded sequence of real or complex numbers. The natural approach is to consider the maximal operator

$$
\mathcal{T}_{\varphi}^{*} f(x)=\sup _{N}\left|\mathcal{T}_{N, \varphi} f(x)\right|,
$$

where for each $N=\left(N_{1}, N_{2}\right) \in \mathbb{Z}^{2}$ with $N_{1} \leq N_{2}, \mathcal{T}_{N, \varphi}$ is the truncation operator 


$$
\mathcal{T}_{N, \varphi} f(x)=\sum_{k=N_{1}}^{N_{2}} v_{k}\left(\mathcal{A}_{\varepsilon_{k}, \varphi} f(x)-\mathcal{A}_{\varepsilon_{k-1}, \varphi} f(x)\right) .
$$

Unlike the problem of the boundedness of $\mathcal{M}_{\varphi}^{+}$, it is not immediately clear how to estimate the maximal operator $\mathcal{T}_{\varphi}^{*}$ by $\mathcal{T}^{*}$, i.e., by the corresponding operator associated to the standard ergodic averages, or, in other words, it is not obvious how to reduce the general problem of the convergence of $(1.8)$ to the case $\varphi=\chi_{(0,1)}$. That is due to the nature of the operator $\mathcal{T}_{\varphi}^{*}$ which is essentially a singular integral maximal operator (when we look at the real line with $\tau_{t} x=x+t$ ).

Our results are collected in the following theorems.

Theorem 1.9. Let $(X, \mathcal{F}, \nu), \Gamma,\left\{g_{t}: t \in \mathbb{R}\right\}, \mathcal{G}$ and $\varphi$ be as in Theorem 1.7. Let $1 \leq p<\infty$ and assume that the semigroup $\mathcal{G}_{+}$is Cesàro bounded in $L^{p}(\nu)$.

(a) If $1<p<\infty$ then there exists $C>0$ such that for all $f \in L^{p}(\nu)$, $\int_{X}\left|\mathcal{T}_{\varphi}^{*} f\right|^{p} d \nu \leq C \int_{X}|f|^{p} d \nu$

(b) If $p=1$ and $g_{t}(x)=1$ then there exists $C>0$ such that for all $f \in$ $L^{1}(\nu)$ and all $\lambda>0, \nu\left(\left\{x \in X:\left|\mathcal{T}_{\varphi}^{*} f(x)\right|>\lambda\right\}\right) \leq(C / \lambda) \int_{X}|f| d \nu$.

To obtain the a.e. convergence of $\mathcal{T}_{N, \varphi} f$ it suffices to prove that the limits $\lim _{N \rightarrow \infty} \mathcal{T}_{N, \varphi}^{1} f(x)$ and $\lim _{N \rightarrow \infty} \mathcal{T}_{N, \varphi}^{2} f(x)$ exist a.e., where

$$
\begin{aligned}
& \mathcal{T}_{N, \varphi}^{1} f(x)=\sum_{k=-N}^{0} v_{k}\left(\mathcal{A}_{\varepsilon_{k}, \varphi}^{+} f(x)-\mathcal{A}_{\varepsilon_{k-1}, \varphi}^{+} f(x)\right), \\
& \mathcal{T}_{N, \varphi}^{2} f(x)=\sum_{k=1}^{N} v_{k}\left(\mathcal{A}_{\varepsilon_{k}, \varphi}^{+} f(x)-\mathcal{A}_{\varepsilon_{k-1}, \varphi}^{+} f(x)\right) .
\end{aligned}
$$

(Here $N$ stands for a natural number.) We shall need some extra assumptions on $\varphi$ but we point out that the examples in the introduction and others as the Poisson kernel, $\varphi(t)=1 /\left(1+t^{2}\right)$, satisfy these conditions.

Theorem 1.10. Let $(X, \mathcal{F}, \nu), \Gamma,\left\{g_{t}: t \in \mathbb{R}\right\}, \mathcal{G}$ and $\varphi$ be as in Theorem 1.7. Let $1<p<\infty$. Assume that $\int t^{\beta} \varphi(t) d t<\infty$ for all $0<\beta<1$ and the semigroup $\mathcal{G}_{+}$is Cesàro bounded in $L^{p}(\nu)$. Then for all $f \in L^{p}(\nu)$, the sequence $\mathcal{T}_{N, \varphi}^{1} f$ converges a.e. and in $L^{p}(\nu)$ as $N \rightarrow \infty$.

If $g_{t}(x)=1$, that is, $T^{t} f(x)=f\left(\tau_{t} x\right)$, we can prove the same result, including the case $p=1$, with a weaker condition on $\varphi$. The key point is that in this case the operators $T^{t}$ are contractions in $L^{\infty}(\nu)$.

TheOREM 1.11. Let $(X, \mathcal{F}, \nu), \Gamma$ and $\varphi$ be as in Theorem 1.7. Let $\mathcal{G}$ be the group defined as $T^{t} f(x)=f\left(\tau_{t} x\right)$. Let $1 \leq p<\infty$. Assume that $\int_{1}^{\infty}|\log t| \varphi(t) d t<\infty$ and the semigroup $\mathcal{G}_{+}$is Cesàro bounded in $L^{p}(\nu)$. 
(a) If $1<p<\infty$ then for all $f \in L^{p}(\nu)$, the sequence $\mathcal{T}_{N, \varphi}^{1} f$ converges a.e. and in $L^{p}(\nu)$ as $N \rightarrow \infty$.

(b) If $p=1$ then for all $f \in L^{1}(\nu)$, the sequence $\mathcal{T}_{N, \varphi}^{1} f$ converges a.e. and in measure as $N \rightarrow \infty$.

For the same class of semigroups, i.e., $g_{t}(x)=1$, we have the following result for the convergence of $\mathcal{T}_{N, \varphi}^{2} f$.

TheOREM 1.12. Let $(X, \mathcal{F}, \nu), \Gamma$ and $\varphi$ be as in Theorem 1.7. Let $\mathcal{G}$ be the group defined as $T^{t} f(x)=f\left(\tau_{t} x\right)$. Let $1 \leq p<\infty$. Assume that $\int_{0}^{1}|\log t| \varphi(t) d t<\infty$ and the semigroup $\mathcal{G}_{+}$is Cesàro bounded in $L^{p}(\nu)$.

(a) If $1<p<\infty$ then for all $f \in L^{p}(\nu)$, the sequence $\mathcal{T}_{N, \varphi}^{2} f$ converges a.e. and in $L^{p}(\nu)$ as $N \rightarrow \infty$.

(b) If $p=1$ then for all $f \in L^{1}(\nu)$, the sequence $\mathcal{T}_{N, \varphi}^{2} f$ converges a.e. and in measure as $N \rightarrow \infty$.

Under the assumptions in Theorems 1.11 and 1.12 , if $\int_{0}^{\infty}|\log t| \varphi(t) d t$ $<\infty$ and the semigroup $\mathcal{G}_{+}$is Cesàro bounded in $L^{p}(\nu)$ then the corresponding results on convergence of $\mathcal{T}_{N, \varphi} f$ hold as $N=\left(N_{1}, N_{2}\right) \rightarrow(-\infty, \infty)$.

For general groups but for standard averages, we can obtain the a.e. convergence of $\mathcal{T}_{N, \varphi}^{2} f$.

Theorem 1.13. Let $(X, \mathcal{F}, \nu), \Gamma,\left\{g_{t}: t \in \mathbb{R}\right\}$ and $\mathcal{G}$ be as in Theorem 1.7. Let $1<p<\infty$ and assume that the semigroup $\mathcal{G}_{+}$is Cesàro bounded in $L^{p}(\nu)$. Then for all $f \in L^{p}(\nu)$, the sequence $\mathcal{T}_{N}^{2} f$ converges a.e. and in $L^{p}(\nu)$ as $N \rightarrow \infty$.

Applying Theorems 1.10 and 1.13 we obtain the following result for general groups and standard averages.

TheOREM 1.14. Let $(X, \mathcal{F}, \nu), \Gamma,\left\{g_{t}: t \in \mathbb{R}\right\}$ and $\mathcal{G}$ be as in Theorem 1.7. Let $1<p<\infty$ and assume that the semigroup $\mathcal{G}_{+}$is Cesàro bounded in $L^{p}(\nu)$. Then, for all $f \in L^{p}(\nu)$, the sequence $\mathcal{T}_{N} f$ converges a.e. and in $L^{p}(\nu)$ as $N=\left(N_{1}, N_{2}\right) \rightarrow(-\infty, \infty)$.

REMARK 1.15. As Jones and Rosenblatt remarked in [14], once we have Theorem 1.9, we can deduce, under the same assumptions, that if $1<p$ $<\infty$ then the square operator $S f(x)=\left(\sum_{k=-\infty}^{\infty}\left|\mathcal{A}_{\varepsilon_{k}, \varphi} f-\mathcal{A}_{\varepsilon_{k-1}, \varphi} f\right|^{2}\right)^{1 / 2}$ is bounded in $L^{p}(\nu)$. Furthermore, as in [14], Theorem 1.9 implies that the series

$$
\sum_{k=-\infty}^{\infty}\left(\mathcal{A}_{\varepsilon_{k}, \varphi} f(x)-\mathcal{A}_{\varepsilon_{k-1}, \varphi} f(x)\right)
$$

converges unconditionally in $L^{p}(\nu), 1<p<\infty$. We remark that Johnson 13. characterized the decreasing sequences $\varepsilon_{k}$ with $\lim _{k \rightarrow-\infty} \varepsilon_{k}=0$ such that the series $\sum_{k=-\infty}^{0}\left(\mathcal{A}_{\varepsilon_{k}, \varphi} f(x)-\mathcal{A}_{\varepsilon_{k-1}, \varphi} f(x)\right)$ is unconditionally conver- 
gent for all $f \in L^{2}(\nu)$ in the case $X=[0,1)$ with $\tau_{t}(x)=x+t(\bmod 1)$ and $\varphi=\chi_{(0,1)}$. This characterization allows one to see that the lacunary sequences are good but $\varepsilon_{k}=-1 / k, k<0$, is a bad sequence, in the sense that the series does not converge unconditionally for all $f \in L^{2}(\nu)$ (see [13]). Therefore, our results are not valid for $\varepsilon_{k}=-1 / k, k<0$, and we have to restrict our attention to a class of subsequences, for instance, the lacunary sequences, as we have done.

We start by establishing in $\S 2$ the results for $\mathcal{T}_{\varphi}^{*}$ in the case of the real line and $\tau_{t} x=x+t$ in weighted $L^{p}$ spaces. We prove Theorem 1.7 in $\S 3$. Next, we transfer the results in $\S 2$ to the ergodic setting and prove Theorem 1.9 in $\S 4$. The proofs of Theorems 1.101 .13 are in $\S 5$. We point out that in $\S 3$ and $\S 4$ we need the results in weighted spaces. One of the difficulties in the transference argument in $\S 4$ comes from the fact that $\varphi$ does not necessarily have compact support. Another problem to overcome is the a.e. convergence of the truncation operators $\mathcal{T}_{N, \varphi} f$ for functions $f$ in the suitable dense class. Finally, in $\S 6$, we provide general examples of Cesàro bounded semigroups.

2. Theorem 1.9 on the real line for the translation flow. Let us consider $X=\mathbb{R}$ with the Lebesgue measure, the flow on $\mathbb{R}$ defined by $\tau_{t}(x)=x+t$ and $g_{t}(x)=1$. Let $\varphi$ be a nonnegative integrable decreasing function on $(0, \infty)$. The $\varphi$-averages associated to this flow are

$$
A_{\varepsilon, \varphi}^{+} f(x)=\frac{1}{\varepsilon} \int_{0}^{\infty} f(x+t) \varphi(t / \varepsilon) d t .
$$

In this section we consider $\varphi$ extended to the whole real line with $\varphi(t)=0$ for $t \leq 0$ and we define $\tilde{\varphi}(t)=\varphi(-t)$. With this notation,

$$
A_{\varepsilon, \varphi}^{+} f(x)=\frac{1}{\varepsilon} \int_{-\infty}^{\infty} f(x-t) \tilde{\varphi}(t / \varepsilon) d t=f * \tilde{\varphi}_{\varepsilon}(x) .
$$

Notice that $\tilde{\varphi}$ is increasing in $(-\infty, 0)$. It is well known (use Proposition 1.3 that the maximal function $M_{\varphi} f(x)=\sup _{\varepsilon>0}\left|A_{\varepsilon, \varphi}^{+} f(x)\right|$ is controlled by the one-sided Hardy-Littlewood maximal function $M^{+} f(x)=$ $\sup _{\varepsilon>0} \varepsilon^{-1} \int_{0}^{\varepsilon}|f(x+t)| d t$. More precisely,

$$
M_{\varphi} f(x) \leq\left(\int_{-\infty}^{\infty} \varphi\right) M^{+} f(x) .
$$

(Notice that $M^{+}$is $M_{\varphi}$ for $\varphi=\chi_{(0,1)}$.) It follows that weights good for $M^{+}$ are also good for $M_{\varphi}$ (by a weight we mean a nonnegative measurable function defined on $\mathbb{R}$ ). The following results can be obtained from the theorems in [24] and [20]. 
(1) Assume that $w \in A_{1}^{+}$, i.e., there exists $C$ such that

$$
M^{-} w(x)=\sup _{\varepsilon>0} \frac{1}{\varepsilon} \int_{0}^{\varepsilon}|w(x-t)| d t \leq C w(x) \quad \text { a.e. }
$$

( $M^{-}$is the left-sided Hardy-Littlewood maximal function). Then the operator $M_{\varphi}$ is of weak type $(1,1)$ with respect to the measure $w(x) d x$, that is, there exists $C$ such that $\int_{\left\{x: M_{\varphi} f(x)>\lambda\right\}} w \leq$ $(C / \lambda) \int|f| w$ for all $\lambda>0$ and all $f \in L^{1}(w)$.

(2) Assume that $w \in A_{p}^{+}$, i.e., there exists $C$ such that for any three points $a<b<c$,

$$
\left(\int_{a}^{b} w\right)^{1 / p}\left(\int_{b}^{c} w^{1-p^{\prime}}\right)^{1 / p^{\prime}} \leq C(c-a)
$$

where $p+p^{\prime}=p p^{\prime}$. Then the operator $M_{\varphi}$ is bounded in $L^{p}(w), 1<$ $p<\infty$, that is, there exists $C>0$ such that $\int\left|M_{\varphi} f\right|^{p} w \leq C \int|f|^{p} w$ for all $f \in L^{p}(w)$.

Remarks 2.1. Notice that $w \in A_{p}^{+}, 1<p<\infty$, if and only if 2.1 holds for $a<b<c$ with $b=(a+c) / 2$. We point out that we can define $A_{p}^{-}$ classes, reversing the orientation of the real line, and obtain the corresponding results for the maximal function associated to a function $\varphi$ supported in $(-\infty, 0)$. There are many important properties of these classes of weights; in particular, in $\$ 6$ we shall frequently use the following result (see 24] and [20]): $w \in A_{p}^{+}$if and only if there exist $u \in A_{1}^{+}$and $v \in A_{1}^{-}$such that $w=u v^{1-p}$.

Throughout the paper, we will consider a bounded sequence $v=\left\{v_{k}\right\}$, $k \in \mathbb{Z}$, of real or complex numbers and a lacunary sequence $\varepsilon=\left\{\varepsilon_{k}\right\}$ of positive numbers. We say that $v=\left\{v_{k}\right\}$ is a multiplying sequence and we write $\|v\|_{\infty}=\sup _{k}\left|v_{k}\right|$. For each $N \in \mathbb{Z}^{2}, N=\left(N_{1}, N_{2}\right)$ with $N_{1} \leq N_{2}$, we define the sum

$$
T_{N, \varphi} f(x)=\sum_{k=N_{1}}^{N_{2}} v_{k}\left(A_{\varepsilon_{k}, \varphi}^{+} f(x)-A_{\varepsilon_{k-1}, \varphi}^{+} f(x)\right)=K_{N, \varphi} * f(x),
$$

where $K_{N, \varphi}(x)=\sum_{k=N_{1}}^{N_{2}} v_{k}\left(\tilde{\varphi}_{\varepsilon_{k}}(x)-\tilde{\varphi}_{\varepsilon_{k-1}}(x)\right)$. Notice that $T_{N, \varphi}$ is the operator $\mathcal{T}_{N, \varphi}$ defined in the previous section with the flow $\tau_{t}(x)=x+t$ and $g_{t}(x)=1$. If we need to emphasize the dependence on $\varepsilon=\left\{\varepsilon_{k}\right\}$ and $v=\left\{v_{k}\right\}$ we shall write $T_{N, \varphi, \varepsilon, v}$ and $K_{N, \varphi, \varepsilon, v}$. As usual, to prove the a.e. convergence, we shall study the boundedness of the associated maximal operator

$$
T_{\varphi}^{*} f(x)=\sup _{N \in \mathbb{Z}^{2}}\left|T_{\varphi, N} f(x)\right|
$$


in the setting of the weighted spaces $L^{p}(w)=\left\{f:\left(\int_{\mathbb{R}}|f|^{p} w\right)^{1 / p}<\infty\right\}$. (If necessary, we shall write $T_{\varphi, \varepsilon, v}^{*}$.) Since the operators $T_{\varphi, N}$ are convolution operators with kernels $K_{\varphi, N}$ supported in $(-\infty, 0)$, the study of $T_{\varphi}^{*}$ and $T_{\varphi, N}$ is related to the right-sided Hardy-Littlewood maximal operator $M^{+}$.

Now we can state the main result in this section.

THEOREM 2.2. Let $\varphi$ be a nonnegative integrable function with support in $(0, \infty)$ and decreasing in that interval. Let $\varepsilon=\left\{\varepsilon_{k}\right\}$ be a $\rho$-lacunary sequence and let $v=\left\{v_{k}\right\}$ be a multiplying sequence.

(i) If $1<p<\infty$ and $w \in A_{p}^{+}$then there exists a constant $C$ depending only on $\rho, p,\|v\|_{\infty}$ and $w$ such that $\int_{\mathbb{R}}\left|T_{\varphi}^{*} f\right|^{p} w \leq C\left(\int \varphi\right)^{p} \int_{\mathbb{R}}|f|^{p} w$ for all $f \in L^{p}(w)$.

(ii) If $w \in A_{1}^{+}$then there exists $C$ depending only on $\rho,\|v\|_{\infty}$ and $w$ such that $\int_{\left\{x \in \mathbb{R}:\left|T_{\varphi}^{*} f(x)\right|>\lambda\right\}} w \leq(C / \lambda)\left(\int \varphi\right)\|f\|_{L^{1}(w)}$ for all $\lambda>0$ and all $f \in L^{1}(w)$.

The organization of this section is as follows. Subsection 2.1 is devoted to notations and properties of lacunary sequences. In Subsections 2.2 and 2.3 we prove Theorem 2.2(i) and Theorem 2.2(ii) respectively.

Throughout this paper, we shall use the notations introduced in this section and the letter $C$ will mean a positive constant not necessarily the same at each occurrence.

2.1. Lacunary sequences. In this section we establish in this section some properties of the $\rho$-lacunary sequence $\varepsilon=\left\{\varepsilon_{k}\right\}$. The next proposition shows that, without loss of generality, we may assume that

$$
1<\rho \leq \varepsilon_{k+1} / \varepsilon_{k} \leq \rho^{2} .
$$

Proposition 2.3. Given the $\rho$-lacunary sequence $\varepsilon=\left\{\varepsilon_{k}\right\}$ and the multiplying sequence $v=\left\{v_{k}\right\}$, we can define a $\rho$-lacunary sequence $\eta=\left\{\eta_{k}\right\}$ and a multiplying sequence $u=\left\{u_{k}\right\}$ with the following properties:

(i) $1<\rho \leq \eta_{k+1} / \eta_{k} \leq \rho^{2}$ and $\|v\|_{\infty}=\|u\|_{\infty}$.

(ii) For all $N=\left(N_{1}, N_{2}\right)$ there exists $M=\left(M_{1}, M_{2}\right)$ with $T_{N, \varphi}=\widetilde{T}_{M, \varphi}$, where $\widetilde{T}_{M, \varphi}$ is the operator defined in 2.2 for $\eta=\left\{\eta_{k}\right\}$ and $u=$ $\left\{u_{k}\right\}$.

The proof is exactly as in the case $\varphi=\chi_{(0,1)}$ (see [3]). It follows from this proposition that it is enough to prove all the results of this paper in the case of a $\rho$-lacunary sequence satisfying (2.3). For this reason, in the rest of the paper we tacitly assume that $\left\{\varepsilon_{k}\right\}$ satisfies (2.3). Observe that, under this assumption,

$$
(1 / \rho)^{2(m-n)} \leq \varepsilon_{n} / \varepsilon_{m} \leq(1 / \rho)^{m-n} \quad \text { for all } m>n .
$$


If we denote by $\alpha$ the smallest positive integer such that $1 / \rho+(1 / \rho)^{\alpha} \leq 1$, from (2.4) we get $\varepsilon_{i}+\varepsilon_{m} \leq \varepsilon_{m+1}$ for all $m \geq i+\alpha-1$.

2.2. Proof of Theorem 2.2(i). First assume that $\varphi$ is simple, that is, $\varphi=\sum_{\ell=1}^{s} a_{\ell} \chi_{\left(-b_{\ell}, 0\right)}, a_{\ell} \geq 0$. We point out that this theorem was proved in [3] for the function $\chi=\chi_{(-1,0)}$. Observe that the kernels $K_{N, \varphi}$ satisfy

$$
K_{N, \varphi}=\sum_{\ell=1}^{s} a_{\ell} b_{\ell} \sum_{k=N_{1}}^{N_{2}} v_{k}\left(\frac{1}{b_{\ell} \varepsilon_{k}} \chi_{\left(-b_{\ell} \varepsilon_{k}, 0\right)}-\frac{1}{b_{\ell} \varepsilon_{k-1}} \chi_{\left(-b_{\ell} \varepsilon_{k-1}, 0\right)}\right) .
$$

For each $\ell$, set $\varepsilon^{\ell}=\left\{\varepsilon_{k}^{\ell}\right\}$ where $\varepsilon_{k}^{\ell}=b_{\ell} \varepsilon_{k}$ is a $\rho$-lacunary sequence and the equality can be written as $K_{N, \varphi}=\sum_{\ell=1}^{s} a_{\ell} b_{\ell} K_{N, \chi, \varepsilon^{\ell}, v}$. Therefore, $T_{N, \varphi}=$ $\sum_{\ell=1}^{s} a_{\ell} b_{\ell} T_{N, \chi, \varepsilon^{\ell}, v}$ and $T_{\varphi}^{*} \leq \sum_{\ell=1}^{s} a_{\ell} b_{\ell} T_{\chi, \varepsilon^{\ell}, v}^{*}$. By the results in [3], there exists a constant $C$ depending only on $\rho$ and $\|v\|_{\infty}$ such that $\int\left|T_{\chi, \varepsilon^{\ell}, v}^{*} f\right|^{p} w \leq$ $C \int|f|^{p} w$. Thus

$$
\int\left|T_{\varphi}^{*} f\right|^{p} w \leq C\left(\sum_{\ell=1}^{s} a_{\ell} b_{\ell}\right)^{p} \int|f|^{p} w=C\left(\int \varphi\right)^{p} \int|f|^{p} w .
$$

Now, let $f \in L^{1}(d x) \cap L^{p}(w)$. Choose a sequence $\left\{\varphi_{k}\right\}$ of simple functions with support in $(0, \infty)$, decreasing in $(0, \infty)$ and such that $\varphi_{k}$ converges to $\varphi$ in the $L^{1}$-norm. Then $T_{N, \varphi_{k}} f$ converges to $T_{N, \varphi} f$ in the $L^{1}$-norm as $k \rightarrow \infty$. Fix a positive integer $M$. It follows that there exists a subsequence $\varphi_{k_{j}}$ such that $T_{N, \varphi_{k_{j}}} f$ converges a.e. to $T_{N, \varphi} f$ as $j \rightarrow \infty$ for all $N=\left(N_{1}, N_{2}\right)$ such that $\left|N_{1}\right|,\left|N_{2}\right| \leq M$. Then $\left|T_{N, \varphi} f\right| \leq \liminf _{j \rightarrow \infty} T_{\varphi_{k_{j}}}^{*} f$ almost everywhere for all $N=\left(N_{1}, N_{2}\right)$ such that $\left|N_{1}\right|,\left|N_{2}\right| \leq M$. Consequently, $\sup _{N=\left(N_{1}, N_{2}\right):\left|N_{1}\right|,\left|N_{2}\right| \leq M}\left|T_{N, \varphi} f\right| \leq \liminf _{j \rightarrow \infty} T_{\varphi_{k_{j}}}^{*} f$, a.e. By the Fatou Lemma and the result for simple functions we have

$$
\begin{aligned}
\int_{N=\left(N_{1}, N_{2}\right):\left|N_{1}\right|,\left|N_{2}\right| \leq M}\left|T_{N, \varphi} f\right|^{p} w \leq \liminf _{j \rightarrow \infty} \int\left|T_{\varphi_{k_{j}}}^{*} f\right|^{p} w \\
\leq C \liminf _{j \rightarrow \infty}\left(\int \varphi_{k_{j}}\right)^{p} \int|f|^{p} w=C\left(\int \varphi\right)^{p} \int|f|^{p} w .
\end{aligned}
$$

Letting $M$ tend to $\infty$ we obtain $\int\left|T_{\varphi}^{*} f\right|^{p} w \leq C\left(\int \varphi\right)^{p} \int|f|^{p} w$ for $f \in L^{1}(d x) \cap$ $L^{p}(w)$. Now let $f \in L^{p}(w)$. There exists a sequence $f_{n} \in L^{1}(d x) \cap L^{p}(w)$ that converges to $f$ in $L^{p}(w)$. Fix $M$. There is a constant $C_{M}$ such that if $N=\left(N_{1}, N_{2}\right)$ with $\left|N_{1}\right|,\left|N_{2}\right| \leq M$, then

$$
\left|T_{N, \varphi} f\right| \leq\left|T_{N, \varphi}\left(f-f_{n}\right)\right|+\left|T_{N, \varphi}\left(f_{n}\right)\right| \leq C_{M} M^{+}\left(f-f_{n}\right)+T_{\varphi}^{*} f_{n} .
$$

Therefore $\sup _{N=\left(N_{1}, N_{2}\right):\left|N_{1}\right|,\left|N_{2}\right| \leq M}\left|T_{N, \varphi} f\right| \leq C_{M} M^{+}\left(f-f_{n}\right)+T^{*} f_{n}$. Using that $w \in A_{p}^{+}$and what we have already proved, we have 


$$
\begin{aligned}
\int_{N=\left(N_{1}, N_{2}\right):\left|N_{1}\right|,\left|N_{2}\right| \leq M}\left|T_{N, \varphi} f\right|^{p} w & \leq C_{M} \int\left|M^{+}\left(f-f_{n}\right)\right|^{p} w+C \int\left|T_{\varphi}^{*} f_{n}\right|^{p} w \\
& \leq C_{M} \int\left|f-f_{n}\right|^{p} w+C\left(\int \varphi\right)^{p} \int\left|f_{n}\right|^{p} w .
\end{aligned}
$$

Letting $n \rightarrow \infty$ and then $M \rightarrow \infty$ we finish the proof.

2.3. Proof of Theorem 2.2(ii). As in [3] for the function $\chi=\chi_{(-1,0)}$, the theorem follows from Theorem 2.2(i) and the following lemma.

LEMma 2.4. Let a be supported on $I=\left(x^{*}, x^{*}+h\right)$ with $\int_{I} a=0$ and let $w \in A_{1}^{+}$. If $A=\rho^{2(\alpha+1)}$ there exists $C$, independent of $x^{*}, h$ and $a$, such that

$$
\int_{z<x^{*}-A h} T_{\varphi}^{*} a(z) w(z) d z \leq C \int_{I}|a(z)| w(z) d z .
$$

Proof. We start by pointing out that this result was proved in [3] for the function $\chi=\chi_{(-1,0)}$. Second, as in [3], it suffices to prove

$$
\int_{z<-\varepsilon_{i+\alpha}} T_{\varphi}^{*} a(z) w(z) d z \leq C \int_{I}|a(z)| w(z) d z,
$$

assuming that $I=\left(0, \varepsilon_{i}\right)$.

First we prove the inequality for simple functions. Let $\varphi=\sum_{\ell=1}^{s} a_{\ell} \chi_{\left(-b_{\ell}, 0\right)}$, $a_{\ell} \geq 0$. For each $\ell$, pick $\varepsilon_{t(\ell)}^{\ell}$ such that $\varepsilon_{t(\ell)-1}^{\ell}<\varepsilon_{i} \leq \varepsilon_{t(\ell)}^{\ell}$. Obviously, $a$ has support in $\left(0, \varepsilon_{t(\ell)}^{\ell}\right)$ and $T_{\varphi}^{*} a(z) \leq \sum_{\ell=1}^{s} a_{\ell} b_{\ell} T_{\chi, \varepsilon^{\ell}, v}^{*} a(z)$. Therefore,

$$
\begin{aligned}
\int_{z<-\varepsilon_{i+\alpha}} T_{\varphi}^{*} a(z) w(z) d z \leq & \sum_{\ell=1}^{s} a_{\ell} b_{\ell} \int_{z<-\varepsilon_{i+\alpha}} T_{\chi, \varepsilon^{\ell}, v}^{*} a(z) w(z) d z \\
= & \sum_{\ell=1}^{s} a_{\ell} b_{\ell} \int_{z<-\varepsilon_{t(\ell)+\alpha}^{\ell}} T_{\chi, \varepsilon^{\ell}, v}^{*} a(z) w(z) d z \\
& +\sum_{\ell=1}^{s} a_{\ell} b_{\ell} \int_{-\varepsilon_{t(\ell)+\alpha}^{\ell}<z<-\varepsilon_{i+\alpha}} T_{\chi, \varepsilon^{\ell}, v}^{*} a(z) w(z) d z .
\end{aligned}
$$

By the result in [3] for $\chi=\chi_{(-1,0)}$ (notice that $\varepsilon^{\ell}=\left\{\varepsilon_{k}^{\ell}\right\}$ is a $\rho$-lacunary sequence), we have

$$
\sum_{\ell=1}^{s} a_{\ell} b_{\ell} \int_{z<-\varepsilon_{t(\ell)+\alpha}^{\ell}} T_{\chi, \varepsilon^{\ell}, v}^{*} a(z) w(z) d z \leq C\left(\int \varphi\right) \int|a(z)| w(z) d z .
$$

Now we estimate the term $\sum_{\ell=1}^{s} a_{\ell} b_{\ell} \int_{-\varepsilon_{t(\ell)+\alpha}^{\ell}<z<-\varepsilon_{i+\alpha}} T_{\chi, \varepsilon^{\ell}, v}^{*} a(z) w(z) d z$. Since $\varepsilon_{t(\ell)-1}^{\ell}<\varepsilon_{i}$, we have $\varepsilon_{t(\ell)+\alpha}^{\ell} \leq \varepsilon_{i+2 \alpha+2}$. Therefore 


$$
\int_{-\varepsilon_{t(\ell)+\alpha}^{\ell}<z<-\varepsilon_{i+\alpha}} T_{\chi, \varepsilon^{\ell}, v}^{*} a(z) w(z) d z \leq \sum_{m=i+\alpha}^{i+2 \alpha+1} \int_{-\varepsilon_{m+1}}^{-\varepsilon_{m}} T_{\chi, \varepsilon^{\ell}, v}^{*} a(z) w(z) d z .
$$

Fix $\ell$ and $N \in \mathbb{Z}^{2}$. Notice that $\left|T_{N, \chi, \varepsilon_{k}^{\ell}, v_{k}} a(z)\right|$ is bounded by

$$
\sum_{k}\left|\int_{I} v_{k}\left(\frac{1}{\varepsilon_{k}^{\ell}} \chi_{\left(-\varepsilon_{k}^{\ell}, 0\right)}(z-u)-\frac{1}{\varepsilon_{k-1}^{\ell}} \chi_{\left(-\varepsilon_{k-1}^{\ell}, 0\right)}(z-u)\right) a(u) d u\right| .
$$

If $z \in\left(-\varepsilon_{m+1},-\varepsilon_{m}\right)$ and $u \in I$, then $z-u \in\left(-\varepsilon_{m+2},-\varepsilon_{m}\right)$ and the $k$-terms in the above sum such that $\varepsilon_{k}^{\ell} \leq \varepsilon_{m}$ or $\varepsilon_{m+2} \leq \varepsilon_{k-1}^{\ell}$ are zero: in the first case because $\left(-\varepsilon_{k}^{\ell}, 0\right) \cap\left(-\varepsilon_{m+2},-\varepsilon_{m}\right)=\emptyset$, and in the second case because $\chi_{\left(-\varepsilon_{k}^{\ell}, 0\right)}(z-u)=\chi_{\left(-\varepsilon_{k-1}^{\ell}, 0\right)}(z-u)=1$ and $\int_{I} a=0$. Then we only have at most four terms in the above sum and in these cases $\varepsilon_{k}^{\ell} \approx \varepsilon_{m}$. Consequently, $\left|T_{N, \chi, \varepsilon_{k}^{\ell}, v_{k}} a(z)\right| \leq C\left(1 / \varepsilon_{m}\right) \int_{I}|a(u)| d u$ and

$$
\int_{-\varepsilon_{m+1}}^{-\varepsilon_{m}} T_{\chi, \varepsilon^{\ell}, v}^{*} a(z) w(z) d z \leq C \frac{1}{\varepsilon_{m}} \int_{I}|a(u)| d u \int_{-\varepsilon_{m+1}}^{-\varepsilon_{m}} w(z) d z .
$$

Since $w \in A_{1}^{+}$, we have

$$
\int_{-\varepsilon_{m+1}}^{-\varepsilon_{m}} T_{\chi, \varepsilon^{\ell}, v}^{*} a(z) w(z) d z \leq C \int_{I}|a(u)| w(u) d u .
$$

Hence

$$
\begin{aligned}
\int_{-\varepsilon_{t(\ell)+\alpha}^{\ell}<z<-\varepsilon_{i+\alpha}} T_{\chi, \varepsilon^{\ell}, v}^{*} a(z) w(z) d z & \leq C \sum_{m=i+\alpha}^{i+2 \alpha+1} \int_{I}|a(u)| w(u) d u \\
& =C \int_{I}|a(u)| w(u) d u .
\end{aligned}
$$

Then

$$
\sum_{\ell=1}^{s} a_{\ell} b_{\ell} \int_{-\varepsilon_{t(\ell)+\alpha}^{\ell}<z<-\varepsilon_{i+\alpha}} T_{\chi, \varepsilon^{\ell}, v}^{*} a(z) w(z) d z \leq C\left(\int \varphi\right) \int_{I}|a(u)| w(u) d u,
$$

and we are done for simple functions.

For the general case, using the notations introduced in the proof of Theorem 2.2(i) (with $f=a$ ), we have

$$
\begin{aligned}
\int_{z<-\varepsilon_{i+\alpha}} \sup _{:\left|N_{1}\right|,\left|N_{2}\right| \leq M}\left|T_{N, \varphi} a(z)\right| w(z) d z \leq \liminf _{j \rightarrow \infty} \int_{z<-\varepsilon_{i+\alpha}} T_{\varphi_{k_{j}}}^{*} a(z) w(z) d z \\
\leq C \liminf _{j \rightarrow \infty}\left(\int \varphi_{k_{j}}\right) \int_{I}|a(z)| w(z) d z=C\left(\int \varphi\right) \iint_{I}|a(z)| w(z) d z .
\end{aligned}
$$

Letting $M$ tend to $\infty$, we are done. 


\section{Proof of Theorem 1.7}

Proof of (a)(ii) and (b)(ii) for $\varphi=\chi_{(0,1)}$. We follow the idea in [16] (see Theorem 2.5 on page 11). Since the semigroup is Cesàro bounded in $L^{p}(\nu)$, for all $f \in L^{p}(\nu)$ and a.e. $x \in X$ the functions $f^{x}(s)=T^{s} f(x)$ are locally integrable. It follows that for a.e. $x$,

$$
\lim _{\varepsilon \rightarrow 0^{+}} \frac{1}{\varepsilon} \int_{0}^{\varepsilon} f^{x}(s+t) d t=f^{x}(s) \text { a.e. } s .
$$

Since, for a.e. $s$ and a.e. $t, f^{x}(s+t)=g_{s}(x) g_{t}\left(\tau_{s} x\right) f\left(\tau_{s+t} x\right)$ and $f^{x}(s)=$ $g_{s}(x) f\left(\tau_{s} x\right)$, we have

$$
\lim _{\varepsilon \rightarrow 0^{+}} \frac{1}{\varepsilon} \int_{0}^{\varepsilon} g_{t}\left(\tau_{s} x\right) f\left(\tau_{s+t} x\right) d t=f\left(\tau_{s} x\right) \quad \text { a.e. } s \geq 0 .
$$

Let $E=\left\{(x, s): s \geq 0, \lim _{\varepsilon \rightarrow 0^{+}} \varepsilon^{-1} \int_{0}^{\varepsilon} g_{t}\left(\tau_{s} x\right) f\left(\tau_{s+t} x\right) d t=f\left(\tau_{s} x\right)\right\}$. This set is measurable in the product space. Let $N=X \times[0, \infty) \backslash E$ and $N_{x}=\{s \geq 0$ : $(x, s) \in N\}$. Then for almost every $x$, the Lebesgue measure $\left|N_{x}\right|$ is zero. Therefore, $|N|=0$ and for a.e. $s \geq 0$ the set $N^{s}=\{x \in X:(x, s) \in N\}$ has measure zero. Notice that $N^{s}=\tau_{-s}\left(N^{0}\right)$. Let $s>0$ be such that $\nu\left(N^{s}\right)=0$. Since the transformations are nonsingular, we obtain $\nu\left(N^{0}\right)=0$. That means that for a.e. $x, \lim _{\varepsilon \rightarrow 0^{+}} \varepsilon^{-1} \int_{0}^{\varepsilon} g_{t}(x) f\left(\tau_{t} x\right) d t=f(x)$, as we wished to prove.

Before continuing, we notice that we have just proved that $\mathcal{M}^{+} f(x)=$ $\sup _{0<\varepsilon \in \mathbb{Q}} \mathcal{A}_{\varepsilon}^{+}|f|(x)$ a.e. for each measurable function $f$. Therefore $\mathcal{M}^{+} f$ is measurable. It is proved in a similar way that $\mathcal{M}_{\varphi}^{+} f$ is measurable when $\varphi$ is a simple function. Finally, for general $\varphi, \mathcal{M}_{\varphi}^{+} f$ is measurable since it is the limit of $\mathcal{M}_{\varphi_{n}}^{+} f$, where $\varphi_{n}$ is a sequence of simple functions.

Proof of (b)(i). As we said at the beginning of the introduction, the functions $H_{t}(x)$ are measurable with respect to the $\sigma$-algebra product and for a.e. $t$,

$$
\int_{X} f(x) d \nu(x)=\int_{X} f\left(\tau_{t} x\right) H_{t}(x) d \nu(x)
$$

for all nonnegative functions and all $f \in L^{1}(\nu)$ (see $(1.3)$ and keep in mind that we assume $g_{t}(x)=1$ in the case $\left.p=1\right)$. Since the flow is Cesàro bounded in $L^{1}(\nu)$, by Tonelli's Theorem we have

$$
\frac{1}{\varepsilon} \int_{0}^{\varepsilon} \int_{X} f\left(\tau_{t} x\right) d \nu d t \leq C \int_{X} f(x) d \nu
$$

for every $\varepsilon>0$ and each measurable function $f \geq 0$. But, for almost all $t$, $\int_{X} f\left(\tau_{t} x\right) d \nu=\int_{X} f(x) H_{-t}(x) d \nu$. Therefore 


$$
\int_{X} f(x)\left(\frac{1}{\varepsilon} \int_{0}^{\varepsilon} H_{-t}(x) d t\right) d \nu \leq C \int_{X} f(x) d \nu
$$

for all nonnegative measurable functions $f$, which implies $\varepsilon^{-} \int_{0}^{\varepsilon} H_{-t}(x) d t$ $\leq C$ a.e. $x$. Since the function on the left-hand side is $\mathcal{F}$-measurable and $\tau_{s}$ is nonsingular we see that for all $s, \varepsilon^{-1} \int_{0}^{\varepsilon} H_{-t}\left(\tau_{s} x\right) d t \leq C$ a.e. $x$. Multiplying by $H_{s}(x)$ gives $\varepsilon^{-1} \int_{0}^{\varepsilon} H_{-t}\left(\tau_{s} x\right) H_{s}(x) d t \leq C H_{s}(x)$ a.e. $x$. Therefore, for almost every $s$,

$$
\frac{1}{\varepsilon} \int_{0}^{\varepsilon} H_{-t}\left(\tau_{s} x\right) H_{s}(x) d t=\frac{1}{\varepsilon} \int_{0}^{\varepsilon} H_{s-t}(x) d t \leq C H_{s}(x) \quad \text { a.e. } x .
$$

Notice that the set

$$
E=\left\{(x, s) \in X \times \mathbb{R}: \frac{1}{\varepsilon} \int_{0}^{\varepsilon} H_{-t}\left(\tau_{s} x\right) H_{s}(x) d t>C H_{s}(x)\right\}
$$

is measurable in the completion of the product $\sigma$-algebra $\mathcal{F} \otimes \mathcal{L}$ and the last statement implies that the completion of the product measure of $E$ is zero. Then it follows that for almost every $x \in X$,

$$
\frac{1}{\varepsilon} \int_{s-\varepsilon}^{s} H_{t}(x) d t=\frac{1}{\varepsilon} \int_{0}^{\varepsilon} H_{s-t}(x) d t \leq C H_{s}(x) \text { for a.e. } s,
$$

or, in other words, for almost every $x$ the functions $t \mapsto H_{t}(x)$ satisfy $A_{1}^{+}$ with a constant independent of $x$.

Since $\mathcal{M}_{\varphi}^{+} \leq\left(\int_{0}^{\infty} \varphi\right) \mathcal{M}^{+}$, it is enough to prove the weak type $(1,1)$ inequality for $\mathcal{M}^{+}$and we shall do it by transference arguments. We can assume that $f \geq 0$. For each $\eta>0$, consider $\mathcal{M}_{\eta}^{+} f(x)=\sup _{0<\varepsilon \leq \eta} \mathcal{A}_{\varepsilon}^{+} f(x)$. Let $\lambda>0$ and $E_{\lambda}=\left\{x \in X: \mathcal{M}_{\eta}^{+} f(x)>\lambda\right\}$. Fix $R>0$. Then, by (3.1),

$$
\begin{aligned}
\nu\left(E_{\lambda}\right) & =\frac{1}{R} \int_{0}^{R} \int_{X} \chi_{E_{\lambda}}\left(\tau_{t} x\right) H_{t}(x) d \nu(x) d t \\
& =\int_{X} \frac{1}{R} \int_{0}^{R} \chi_{E_{\lambda}}\left(\tau_{t} x\right) H_{t}(x) d t d \nu(x) .
\end{aligned}
$$

If we define $f^{x}(t)=f\left(\tau_{t} x\right)$, we find that if $R>0, t \leq R$, and $\chi_{E_{\lambda}}\left(\tau_{t} x\right)=1$ then $M^{+}\left(f^{x} \chi_{[0, R+\eta]}\right)(t)>\lambda$. Therefore

$$
\nu\left(E_{\lambda}\right) \leq \int_{X} \frac{1}{R} \int_{\left\{t: M^{+}\left(f^{x} \chi_{[0, R+\eta]}\right)(t)>\lambda\right\}} H_{t}(x) d t d \nu(x) .
$$

Since, for almost every $x$, the functions $t \mapsto H_{t}(x)$ satisfy $A_{1}^{+}$with a constant independent of $x$, and $A_{1}^{+}$characterizes the weak-type $(1,1)$ inequality 
of $M^{+}($see $\S 2)$, we obtain

$$
\begin{aligned}
\nu\left(E_{\lambda}\right) & \leq \frac{C}{\lambda} \int_{X} \frac{1}{R} \int_{0}^{R+\eta} f^{x}(t) H_{t}(x) d t d \nu(x) \\
& =\frac{C}{\lambda R} \int_{0}^{R+\eta} \int_{X} f\left(\tau_{t} x\right) H_{t}(x) d \nu(x) d t \\
& =\frac{C}{\lambda R} \int_{0}^{R+\eta} \int_{X} f(x) d \nu(x) d t=\frac{C(R+\eta)}{\lambda R} \int_{X} f(x) d \nu(x) .
\end{aligned}
$$

Letting $R$ and then $\eta$ tend to infinity we obtain the inequality that we wished to prove.

Proof of $(a)(i)$. We start by proving that our assumption, that the semigroup $\mathcal{G}_{+}$is Cesàro bounded in $L^{p}(\nu)$, implies that

$$
\begin{aligned}
& \text { for almost every } x \in X, \text { the function } t \mapsto H_{t}(x) \\
& \text { belongs to } A_{p}^{+} \text {with a constant independent of } x \text {. }
\end{aligned}
$$

We shall use the ideas of Rubio de Francia about factorization of weights [21]. By hypothesis, there exists $C>0$ independent of $\varepsilon>0$ and $f$ such that

$$
\int_{X}\left|\mathcal{A}_{2 \varepsilon}^{+} f\right|^{p} d \nu \leq C \int_{X}|f|^{p} d \nu \quad \text { for all } f \in L^{p}(\nu),
$$

and consequently

$$
\int_{X}\left|\left(\mathcal{A}_{2 \varepsilon}^{+}\right)^{*} f\right|^{p^{\prime}} d \nu \leq C \int_{X}|f|^{p^{\prime}} d \nu \quad \text { for all } f \in L^{p^{\prime}}(\nu),
$$

where $\left(\mathcal{A}_{2 \varepsilon}^{+}\right)^{*}$ is the adjoint operator of $\mathcal{A}_{2 \varepsilon}^{+} f$. Notice that if $T_{t}^{*}$ is the formal adjoint of $T_{t}$ then for almost all $t$,

$$
\begin{aligned}
T_{t}^{*} h(x) & =\frac{J_{-t}(x)}{g_{-t}(x)} h\left(\tau_{-t} x\right), \\
H_{t}(x) & =\left(\left(T^{-t}\right)^{*} h^{p}\right)(x)\left(T^{t} h^{p^{\prime}}\right)^{1-p}(x) \quad \text { a.e. } x
\end{aligned}
$$

for any function $h>0, h \in L^{p p^{\prime}}(\nu)$. For $h \in L^{p p^{\prime}}(\nu)$, we define $Q_{\varepsilon} h=$ $\left(\mathcal{A}_{2 \varepsilon}^{+}|h|^{p^{\prime}}\right)^{1 / p^{\prime}}$ and $P_{\varepsilon} h=\left(\left(\mathcal{A}_{2 \varepsilon}^{+}\right)^{*}|h|^{p}\right)^{1 / p}$. Then $Q_{\varepsilon}, P_{\varepsilon}$ and $R_{\varepsilon}=Q_{\varepsilon}+P_{\varepsilon}$ are bounded from $L^{p p^{\prime}}(\nu)$ into $L^{p p^{\prime}}(\nu)$ with constants independent of $\varepsilon>0$. Fix $C>0$ such that $\left\|R_{\varepsilon} h\right\|_{L^{p p^{\prime}}(\nu)} \leq C\|h\|_{L^{p p^{\prime}}(\nu)}$ for all $h \in L^{p p^{\prime}}(\nu)$ and all $\varepsilon>0$. For fixed $h>0, h \in L^{p p^{\prime}}(\nu)$, and $\varepsilon>0$, let $G(x)=\sum_{j=0}^{\infty} R_{\varepsilon}^{(j)} h(x) /(2 C)^{j}$, where $R_{\varepsilon}^{(j)}$ is the $j$ th iteration of $R_{\varepsilon}$. Then $G \in L^{p p^{\prime}}(\nu), h \leq G$ a.e., $R_{\varepsilon} G \leq$ $2 C G$ a.e. and so $P_{\varepsilon} G \leq 2 C G$ a.e. and $Q_{\varepsilon} G \leq 2 C G$ a.e., i.e., there exists 
$C>0$ such that

$$
\begin{aligned}
\mathcal{A}_{2 \varepsilon}^{+} G^{p^{\prime}} & \leq C G^{p^{\prime}} \quad \text { a.e. } \\
\left(\mathcal{A}_{2 \varepsilon}^{+}\right)^{*} G^{p} & \leq C G^{p}
\end{aligned}
$$

Since the operators $T^{t}$ are linear and positive, we infer from (3.5) that for $s \leq t \leq s+\varepsilon$,

$$
\begin{aligned}
C T^{t} G^{p^{\prime}}(x) & \geq T^{t}\left(\mathcal{A}_{2 \varepsilon}^{+} G^{p^{\prime}}\right)(x)=\frac{1}{2 \varepsilon} \int_{0}^{2 \varepsilon} T^{t+s} G^{p^{\prime}}(x) d s \\
& =\frac{1}{2 \varepsilon} \int_{t}^{2 \varepsilon+t} T^{u} G^{p^{\prime}}(x) d u \geq \frac{1}{2 \varepsilon} \int_{s+\varepsilon}^{s+2 \varepsilon} T^{u} G^{p^{\prime}}(x) d u .
\end{aligned}
$$

Raising to the power $1-p<0$, multiplying by $\left(T^{-t}\right)^{*} G^{p}(x)$ and using (3.4) we have, for almost all $t$,

$$
C H_{t}(x) \leq\left(\frac{1}{2 \varepsilon} \int_{s+\varepsilon}^{s+2 \varepsilon} T^{u} G^{p^{\prime}}(x) d u\right)^{1-p}\left(T^{-t}\right)^{*} G^{p}(x) \quad \text { a.e. } x
$$

where the exceptional set depends on $\varepsilon$ and $t$. Integrating over any measurable set $A \subset X$ gives

$$
C \int_{A} H_{t}(x) d \nu \leq \int_{A}\left(\frac{1}{2 \varepsilon} \int_{s+\varepsilon}^{s+2 \varepsilon} T^{u} G^{p^{\prime}}(x) d u\right)^{1-p}\left(T^{-t}\right)^{*} G^{p}(x) d \nu
$$

for a.e. $t \in[s, s+\varepsilon]$. Integrating over the interval $[s, s+\varepsilon]$ and applying Fubini's Theorem, we obtain

$$
\begin{aligned}
& C \int_{A} \int_{s}^{s+\varepsilon} H_{t}(x) d t d \nu \\
& \leq \int_{A}\left(\frac{1}{2 \varepsilon} \int_{s+\varepsilon}^{s+2 \varepsilon} T^{u} G^{p^{\prime}}(x) d u\right)^{1-p}\left(\int_{s}^{s+\varepsilon}\left(T^{-t}\right)^{*} G^{p}(x) d t\right) d \nu .
\end{aligned}
$$

Since $A$ is any measurable subset we have

$$
\begin{aligned}
& C \int_{s}^{s+\varepsilon} H_{t}(x) d t \\
& \quad \leq\left(\frac{1}{2 \varepsilon} \int_{s+\varepsilon}^{s+2 \varepsilon} T^{u} G^{p^{\prime}}(x) d u\right)^{1-p}\left(\int_{s}^{s+\varepsilon}\left(T^{-t}\right)^{*} G^{p}(x) d t\right) \quad \text { a.e. } x
\end{aligned}
$$

where the exceptional set depends on $s$ and $\epsilon$. On the other hand, since the adjoints $\left(T^{-t}\right)^{*}$ are also linear and positive, arguing in the same way we 
deduce from (3.6) that

$$
\begin{aligned}
& C \int_{s+\varepsilon}^{s+2 \varepsilon}\left(H_{t}(x)\right)^{1-p^{\prime}} d t \\
& \quad \leq\left(\frac{1}{2 \varepsilon} \int_{s}^{s+\varepsilon}\left(T^{-u}\right)^{*} G^{p}(x) d u\right)^{1-p^{\prime}} \int_{s+\varepsilon}^{s+2 \varepsilon} T^{t} G^{p^{\prime}}(x) d t \quad \text { a.e. } x
\end{aligned}
$$

where the exceptional set depends on $s$ and $\epsilon$. From (3.8) and (3.7), we get

$$
\int_{s}^{s+\varepsilon} H_{t}(x) d t\left(\int_{s+\varepsilon}^{s+2 \varepsilon}\left(H_{t}(x)\right)^{1-p^{\prime}} d t\right)^{p-1} \leq C \varepsilon^{p} \quad \text { a.e. } x
$$

where the exceptional set depends on $s$ and $\epsilon$. Then, for almost every $x$,

$$
\int_{a}^{b} H_{t}(x) d t\left(\int_{b}^{c}\left(H_{t}(x)\right)^{1-p^{\prime}} d t\right)^{p-1} \leq C(c-a)^{p}
$$

for all rational numbers $a<c$ and $b=(a+c) / 2$. Now it is clear that the same holds for all real numbers $a<c$ and $b=(a+c) / 2$. Therefore, (3.2) holds (see Remarks 2.1).

Now, let us prove (a)(i). Since $\mathcal{M}^{+} f(x) \leq \mathcal{M}^{+}(|f|)(x)$, we can assume that $f \geq 0$. For each $\eta>0$, consider $\mathcal{M}_{\eta}^{+} f(x)=\sup _{0<\varepsilon \leq \eta} \mathcal{A}_{\varepsilon}^{+} f(x)$. From the positivity of $T^{t}$ we have $T^{t} \mathcal{M}_{\eta}^{+} f(x)=\mathcal{M}_{\eta}^{+}\left(T^{t} f\right)(x)$. If we define $f^{x}(t)=$ $T^{t} f(x)$, we find for all $R>0$ and all $t \leq R$,

$$
\begin{aligned}
\mathcal{M}_{\eta}^{+}\left(T^{t} f\right)(x) & =\sup _{0<\varepsilon \leq \eta} \frac{1}{\varepsilon} \int_{0}^{\varepsilon} T^{s+t} f(x) d s=\sup _{0<\varepsilon \leq \eta} \frac{1}{\varepsilon} \int_{0}^{\varepsilon} f^{x}(s+t) d s \\
& =\sup _{0<\varepsilon \leq \eta} \frac{1}{\varepsilon} \int_{0}^{\varepsilon} f^{x} \chi_{[0, R+\eta]}(s+t) d s \leq M^{+}\left(f^{x} \chi_{[0, R+\eta]}\right)(t),
\end{aligned}
$$

where $M^{+}$is the one-sided Hardy-Littlewood maximal operator in $\mathbb{R}$. Then, by (1.3), Fubini's Theorem, $(3.2)$ and the fact that $A_{p}^{+}$implies boundedness of the one-sided Hardy-Littlewood maximal operator, we conclude that for each $R>0$,

$$
\begin{aligned}
\int_{X}\left(\mathcal{M}_{\eta}^{+} f(x)\right)^{p} d \nu(x) & =\frac{1}{R} \int_{0}^{R} \int_{X}\left|T^{t} \mathcal{M}_{\eta}^{+} f(x)\right|^{p} H_{t}(x) d \nu(x) d t \\
& \leq \int_{X} \frac{1}{R} \int_{0}^{R}\left|M^{+}\left(f^{x} \chi_{[0, R+\eta]}\right)(t)\right|^{p} H_{t}(x) d t d \nu(x) \\
& \leq \frac{C}{R} \int_{X} \int_{0}^{R+\eta}\left|f^{x}(t)\right|^{p} H_{t}(x) d t d \nu(x)
\end{aligned}
$$




$$
\begin{aligned}
& =\frac{C}{R} \int_{0}^{R+\eta} \int_{X}\left|T^{t} f(x)\right|^{p} H_{t}(x) d \nu(x) d t=\frac{C}{R} \int_{0}^{R+\eta} \int_{X}|f(x)|^{p} d \nu(x) d t \\
& =C \frac{R+\eta}{R} \int_{X}|f(x)|^{p} d \nu(x) .
\end{aligned}
$$

Letting first $R$ and then $\eta$ go to infinity we obtain

$$
\int_{X}\left(\mathcal{M}^{+} f(x)\right)^{p} d \nu(x) \leq C \int_{X}|f(x)|^{p} d \nu(x) .
$$

Proof of (a) (ii) and (b)(ii). Since the maximal operator is bounded in $L^{p}(\nu)(p>1)$ or of weak type $(1,1)$, it is enough to prove the a.e. convergence. By Proposition 1.4 it suffices to show the a.e. convergence in the standard case $\varphi=\chi_{(0,1)}$, which has already been proved at the beginning of this section.

Proof of (a)(iii). Since the maximal operator is bounded in $L^{p}(\nu)$ it is enough to prove the a.e. convergence in a dense class. As before, it is enough to prove it in the standard case $\varphi=\chi_{(0,1)}$. To find the dense class we proceed almost as in Lemma 4.2 of [4]. We shall need some results of independent interest.

LEMMA 3.1. Under the conditions of Theorem 1.7 if $1 \leq r<p$ let $\mathcal{G}_{r}=\left\{S^{t}: t \in \mathbb{R}\right\}$ be the one-parameter group of positive operators defined by $S^{t} f(x)=\left(g_{t}(x)\right)^{r} f\left(\tau_{t} x\right)$ for all $f \geq 0$. Then there exists $r, 1<r<p$, such that the semigroup $\mathcal{G}_{r,+}=\left\{S^{t}: t>0\right\}$ is Cesàro bounded in $L^{p / r}(\nu)$. Furthermore, the maximal operator associated to $\mathcal{G}_{r,+}$ is bounded in $L^{p / r}(\nu)$.

Proof. We have already seen in the proof of $(\mathrm{a})(\mathrm{i})$ that $\mathcal{G}_{+}$Cesàro bounded implies that for almost every $x$ the functions $t \mapsto H_{t}(x)$ belong to $A_{p}^{+}$with a constant independent of $x$ (actually, the implication is an equivalence, see Remark 3.5. Then by the properties of $A_{p}^{+}$classes, there exists $r, 1<r<p$, such that for almost every $x$ the function $t \mapsto H_{t}(x)$ belongs to $A_{p / r}^{+}$with a constant independent of $x$ (see [24] and [19]). We notice that

$$
\int_{X}\left|S^{t} f(x)\right|^{p / r} H_{t}(x) d \nu(x)=\int_{X}|f(x)|^{p / r} d \nu(x)
$$

for all $f \in L^{p / r}(\nu)$ and all $f \geq 0$. Again, by the proof of (a)(i) applied to the semigroup $\mathcal{G}_{r,+}$, the maximal operator associated to $\mathcal{G}_{r,+}$ is bounded in $L^{p / r}(\nu)$, and therefore the semigroup is Cesàro bounded in $L^{p / r}(\nu)$.

LEMMA 3.2. Under the conditions of Theorem 1.7, if $1<p<\infty$, then, for all $f \in L^{p}(\nu)$ :

(a) $\lim _{\varepsilon \rightarrow \infty}\left[\mathcal{A}_{\varepsilon}^{+} f(x)-\mathcal{A}_{\gamma}^{+}\left(\mathcal{A}_{\varepsilon}^{+} f\right)(x)\right]=0$ a.e. for all $\gamma>0$.

(b) $\lim _{\varepsilon \rightarrow \infty}\left\|\mathcal{A}_{\varepsilon}^{+} f-\mathcal{A}_{\gamma}^{+}\left(\mathcal{A}_{\varepsilon}^{+} f\right)\right\|_{p}=0$ for all $\gamma>0$. 
Proof. First, notice that

$$
\mathcal{A}_{\varepsilon}^{+} f(x)-\mathcal{A}_{\gamma}^{+}\left(\mathcal{A}_{\varepsilon}^{+} f\right)(x)=\frac{1}{\gamma} \int_{0}^{\gamma}\left(\mathcal{A}_{\varepsilon}^{+} f(x)-T^{s}\left(\mathcal{A}_{\varepsilon}^{+} f\right)(x)\right) d s .
$$

Fix $0<s<\gamma$ and $\varepsilon>\gamma$. Then

$$
\begin{aligned}
\left|\mathcal{A}_{\varepsilon}^{+} f(x)-T^{s}\left(\mathcal{A}_{\varepsilon}^{+} f\right)(x)\right| & =\left|\frac{1}{\varepsilon} \int_{0}^{\varepsilon} T^{t} f(x) d t-\frac{1}{\varepsilon} \int_{s}^{s+\varepsilon} T^{t} f(x) d t\right| \\
& =\left|\frac{1}{\varepsilon} \int_{0}^{s} T^{t} f(x) d t-\frac{1}{\varepsilon} \int_{\varepsilon}^{s+\varepsilon} T^{t} f(x) d t\right| \\
& \leq \frac{1}{\varepsilon} \int_{0}^{\gamma} T^{t}|f|(x) d t+\frac{1}{\varepsilon} \int_{\varepsilon}^{\gamma+\varepsilon} T^{t}|f|(x) d t .
\end{aligned}
$$

Therefore, by (3.14),

$$
\left|\mathcal{A}_{\varepsilon}^{+} f(x)-\mathcal{A}_{\gamma}^{+}\left(\mathcal{A}_{\varepsilon}^{+} f\right)(x)\right| \leq \frac{1}{\varepsilon} \int_{0}^{\gamma} T^{t}|f|(x) d t+\frac{1}{\varepsilon} \int_{\varepsilon}^{\gamma+\varepsilon} T^{t}|f|(x) d t .
$$

It is clear that $\lim _{\varepsilon \rightarrow \infty} \varepsilon^{-1} \int_{0}^{\gamma} T^{t}|f|(x) d t=0$ for a.e. $x$, since the function $f^{x}(t)=T^{t} f(x)$ is locally integrable for almost every $x$. To control the other term we use Lemma 3.1. Let $p>r>1$, let $\Gamma_{\gamma}=\left\{S^{t}: t \in \mathbb{R}\right\}$ be as in that lemma, let $\widetilde{\mathcal{M}}^{+}$be the maximal operator associated to $\mathcal{G}_{r,+}=\left\{S^{t}: t>0\right\}$. By Lemma 3.1, $\widetilde{\mathcal{M}}^{+}$is bounded from $L^{p / r}(\nu)$ into $L^{p / r}(\nu)$. Consequently, $\widetilde{\mathcal{M}}^{+}\left(|f|^{r}\right)(x)<\infty$ a.e. for $f \in L^{p}(\nu)$. It follows that $\varepsilon^{-1} \int_{\varepsilon}^{\gamma+\varepsilon} T^{t}|f|(x) d t$ tends to 0 a.e. as $\varepsilon$ goes to infinity since

$$
\begin{aligned}
\frac{1}{\varepsilon} \int_{\varepsilon}^{\gamma+\varepsilon} T^{t}|f|(x) d t & \leq \frac{1}{\varepsilon}\left(\int_{\varepsilon}^{\gamma+\varepsilon}\left(T^{t}|f|(x)\right)^{r} d t\right)^{1 / r} \gamma^{1 / r^{\prime}} \\
& \leq \frac{(\gamma+\varepsilon)^{1 / r}}{\varepsilon}\left(\frac{1}{\gamma+\varepsilon} \int_{0}^{\gamma+\varepsilon} S^{t}\left(|f|^{r}\right)(x) d t\right)^{1 / r} \gamma^{1 / r^{\prime}} \\
& \leq \frac{(\gamma+\varepsilon)^{1 / r} \gamma^{1 / r^{\prime}}}{\varepsilon}\left[\widetilde{\mathcal{M}}^{+}\left(|f|^{r}\right)(x)\right]^{1 / r}
\end{aligned}
$$

Therefore (a) is completely proved.

To prove (b) we observe that $\left|\mathcal{A}_{\varepsilon}^{+} f-\mathcal{A}_{\gamma}^{+}\left(\mathcal{A}_{\varepsilon}^{+} f\right)\right| \leq \mathcal{M}^{+} f+\mathcal{M}^{+}\left(\mathcal{A}_{\gamma}^{+} f\right)$. It follows from statement (a)(i) of Theorem 1.7 that $\mathcal{M}^{+} f+\mathcal{M}^{+}\left(\mathcal{A}_{\gamma}^{+} f\right) \in$ $L^{p}(\nu)$. Then (b) follows from (a) and the dominated convergence theorem.

The next theorem follows from Lemma 3.2 using a standard argument. We include it for the sake of completeness. 
TheOREM 3.3. Under the conditions of Theorem 1.7 with $1<p<\infty$, let $A=\left\{f \in L^{p}(\nu): \mathcal{A}_{\gamma}^{+} f=f\right.$ for all $\left.\gamma>0\right\}$ and let $B$ be the linear manifold generated by $\left\{f-\mathcal{A}_{\gamma}^{+} f: f \in L^{p}(\nu), \gamma>0\right\}$. Then $A \oplus \bar{B}=L^{p}(\nu)$, where $\bar{B}$ stands for the closure of $B$ and $A \oplus \bar{B}=\{f+g: f \in A, g \in \bar{B}\}$. In particular $A \oplus B$ is dense in $L^{p}(\nu)$.

Proof. We first prove that $\left\{\mathcal{A}_{\varepsilon}^{+} f\right\}$ is weakly convergent as $\varepsilon$ goes to infinity for all $f \in L^{p}(\nu)$.

Let $f \in L^{p}(\nu)$. By hypothesis, $\sup _{\varepsilon>0}\left\|\mathcal{A}_{\varepsilon}^{+} f\right\|_{L^{p}(\nu)} \leq C\|f\|_{L^{p}(\nu)}$. This shows that the set $\left\{\mathcal{A}_{\varepsilon}^{+} f: \varepsilon>0\right\}$ is bounded in $L^{p}(\nu)$. Therefore there exists a sequence $\left\{\varepsilon_{k}\right\} \rightarrow \infty$ such that $\left\{\mathcal{A}_{\varepsilon_{k}}^{+} f\right\}$ is weakly convergent. If we suppose that $\left\{\mathcal{A}_{\varepsilon}^{+} f\right\}$ is not weakly convergent as $\varepsilon$ goes to infinity, then there exist another sequence $\left\{\eta_{k}\right\} \rightarrow \infty$ and $g_{1}, g_{2} \in L^{p}(\nu), g_{1} \neq g_{2}$, such that $\left\{\mathcal{A}_{\varepsilon_{k}}^{+} f\right\}$ converges weakly to $g_{1}$ and $\left\{\mathcal{A}_{\eta_{k}}^{+} f\right\}$ converges weakly to $g_{2}$. The continuity of $\mathcal{A}_{\gamma}^{+}$gives that $\left\{\mathcal{A}_{\varepsilon_{k}}^{+} f-\mathcal{A}_{\gamma}^{+}\left(\mathcal{A}_{\varepsilon_{k}}^{+} f\right)\right\}$ converges weakly to $g_{1}-\mathcal{A}_{\gamma}^{+} g_{1}$. On the other hand, by Lemma $3.2(\mathrm{~b}),\left\{\mathcal{A}_{\varepsilon_{k}}^{+} f-\mathcal{A}_{\gamma}^{+}\left(\mathcal{A}_{\varepsilon_{k}}^{+} f\right)\right\}$ converges to 0 in $L^{p}(\nu)$. Therefore, $g_{1} \in A$. The same argument shows that $g_{2} \in A$, and so $0 \neq g_{1}-g_{2} \in A$.

We now prove that $g_{1}-g_{2} \in \bar{B}$. Otherwise there exists a linear functional $\Lambda: L^{p}(\nu) \rightarrow \mathbb{R}$ such that $\Lambda(\bar{B})=0$ and $\Lambda\left(g_{1}-g_{2}\right)=1$. It follows that $\Lambda g=\Lambda\left(\mathcal{A}_{\gamma}^{+} g\right)$ for all $g \in L^{p}(\nu)$ and all $\gamma>0$. In particular, $\Lambda\left(\mathcal{A}_{\varepsilon_{k}}^{+} f\right)=\Lambda f$. On the other hand, $\left\{\Lambda\left(\mathcal{A}_{\varepsilon_{k}}^{+} f\right)\right\}$ converges to $\Lambda g_{1}$ in $\mathbb{R}$. Then $\Lambda g_{1}=\Lambda f$. In an analogous way we get $\Lambda g_{2}=\Lambda f$. It follows that $1=\Lambda\left(g_{1}-g_{2}\right)=$ $\Lambda g_{1}-\Lambda g_{2}=0$, a contradiction. Thus $g_{1}-g_{2} \in \bar{B}$.

Let us now prove that $\left\|\mathcal{A}_{\varepsilon}^{+} g\right\|_{L^{p}(\nu)} \rightarrow 0$ as $\varepsilon$ tends to infinity, for all $g \in \bar{B}$. If $g=g_{0}-\mathcal{A}_{\gamma}^{+} g_{0}$ for some $g_{0} \in L^{p}(\nu)$ and $s>0$, this follows from Lemma 3.2 (b), and therefore it holds for any $g \in B$. Now fix $g \in \bar{B}$. For any $\delta>0$, there exists $g_{0} \in B$ such that $\left\|g-g_{0}\right\|_{L^{p}(\nu)}<\delta$. As a consequence,

$$
\begin{aligned}
\left\|\mathcal{A}_{\varepsilon}^{+} g\right\|_{L^{p}(\nu)} & \leq\left\|\mathcal{A}_{\varepsilon}^{+} g-\mathcal{A}_{\varepsilon}^{+} g_{0}\right\|_{L^{p}(\nu)}+\left\|\mathcal{A}_{\varepsilon}^{+} g_{0}\right\|_{L^{p}(\nu)} \\
& =\left\|\mathcal{A}_{\varepsilon}^{+}\left(g-g_{0}\right)\right\|_{L^{p}(\nu)}+\left\|\mathcal{A}_{\varepsilon}^{+} g_{0}\right\|_{L^{p}(\nu)} \leq C \delta+\left\|\mathcal{A}_{\varepsilon}^{+} g_{0}\right\|_{L^{p}(\nu)} .
\end{aligned}
$$

Since $\left\|\mathcal{A}_{\varepsilon}^{+} g_{0}\right\|_{L^{p}(\nu)} \rightarrow 0$ as $\varepsilon$ tends to infinity $\left(g_{0} \in B\right)$ and $\delta$ is any positive number we conclude that $\left\|\mathcal{A}_{\varepsilon}^{+} g\right\|_{L^{p}(\nu)} \rightarrow 0$ as $\varepsilon$ tends to infinity .

We have already seen that $g_{1}-g_{2} \in \bar{B}$. Thus $\left\{\mathcal{A}_{\varepsilon}^{+}\left(g_{1}-g_{2}\right)\right\}$ converges to 0 in $L^{p}(\nu)$. On the other hand, $g_{1}-g_{2} \in A$, which implies that $\mathcal{A}_{\varepsilon}^{+}\left(g_{1}-g_{2}\right)=$ $g_{1}-g_{2}$. Hence $g_{1}-g_{2}=0$, a contradiction. Therefore, $\left\{\mathcal{A}_{\varepsilon}^{+} f\right\}$ is weakly convergent as $\varepsilon$ goes to infinity. (The preceding argument also proves that $A \cap \bar{B}=\{0\}$.

We now prove that $A \oplus \bar{B}=L^{p}(\nu)$. Let $P f$ be the weak limit of $\left\{\mathcal{A}_{\varepsilon}^{+} f\right\}$ as $\varepsilon$ tends to infinity. Then $f=P f+(f-P f)$. From the continuity of $\mathcal{A}_{\gamma}^{+}$and Lemma $3.2(\mathrm{~b})$, it follows that $\mathcal{A}_{\gamma}^{+}(P f)=P f$ for all $\gamma>0$, that is, $P f \in A$. If we suppose that $f-P f \notin \bar{B}$, then there exists a linear 
functional $\Lambda: L^{p}(\nu) \rightarrow \mathbb{R}$ such that $\Lambda(\bar{B})=0$ and $\Lambda(f-P f)=1$. But $P f$ is the weak limit of $\mathcal{A}_{\varepsilon}^{+} f$ and therefore $\Lambda(P f)=\lim _{\varepsilon \rightarrow \infty} \Lambda\left(\mathcal{A}_{\varepsilon}^{+} f\right)$. However, $\Lambda\left(\mathcal{A}_{\varepsilon}^{+} f\right)=\Lambda f$. Therefore $\Lambda(f-P f)=0$, a contradiction.

Now we can conclude the proof of (a)(iii) of Theorem 1.7. Since the maximal operator is bounded in $L^{p}(\nu)$ it is enough to prove the a.e. convergence in the dense class $D_{1}=A \oplus B$. If $f \in A$ this is obvious. For $f \in B$, Lemma 3.2 (a) proves that $\left\{\mathcal{A}_{\varepsilon}^{+} f\right\}$ converges to 0 a.e. as $\varepsilon$ tends to infinity.

REMARK 3.4. The set $A$ in Theorem 3.3 equals $\left\{f \in L^{p}(\nu): T^{s} f=f\right.$ for all $s>0\}$, since it follows from (3.15) and (3.17) that $A_{\varepsilon}^{+} f-T^{s}\left(A_{\varepsilon}^{+} f\right)$ $\rightarrow 0$ a.e. as $\varepsilon \rightarrow \infty$ for all $f \in L^{p}(\nu)$.

Proof of (b)(ii) and (iii). Since the flow is Cesàro bounded in $L^{1}(\nu)$ and $g_{t}(x)=1$, it is Cesàro bounded in $L^{p}(\nu)$ for $1<p<\infty$. Then the averages converge a.e. as $\varepsilon \rightarrow 0$ and as $\varepsilon \rightarrow \infty$ for $f \in L^{p}(\nu) \cap L^{1}(\nu)$, which is a dense set in $L^{1}(\nu)$. Together with the weak type $(1,1)$ inequality for $\mathcal{M}^{+}$this gives the almost everywhere convergence and the convergence in measure of the $\varphi$-averages for all $f \in L^{1}(\nu)$.

REMARK 3.5. It follows from the proof of Theorem 1.7 that the semigroup $\mathcal{G}_{+}$is Cesàro bounded in $L^{p}(\nu)$ if and only if

$$
\begin{aligned}
& \text { for almost every } x \in X \text {, the function } t \mapsto H_{t}(x) \\
& \text { belongs to } A_{p}^{+} \text {with a constant independent of } x,
\end{aligned}
$$

where $H_{t}(x)$ is defined in 1.3 .

4. Proof of Theorem 1.9. We shall use transference arguments and only prove (a) since the proof of (b) is similar. We point out that the support of $\varphi$ is not necessarily bounded. For that reason, we have to modify slightly the usual transference arguments. As before, we shall use the notation $f^{x}(t)=T^{t} f(x)$.

For each natural $M$, we consider the set $Q_{M}=\left\{N \in \mathbb{Z}^{2}: N=\right.$ $\left.\left(N_{1}, N_{2}\right), N_{1} \leq N_{2},\left|N_{1}\right| \leq M,\left|N_{2}\right| \leq M\right\}$ and the operator $\mathcal{T}_{\varphi, M}^{*} f(x)=$ $\sup _{N \in Q_{M}}\left|\mathcal{T}_{\varphi, N} f(x)\right|$.

Let $L>0$ and $\varphi_{L}=\varphi \chi_{(0, L]}$. Then $\mathcal{T}_{\varphi, M}^{*} \leq \mathcal{T}_{\varphi_{L}, M}^{*}+\mathcal{T}_{\varphi-\varphi_{L}, M}^{*}$. Since the semigroup $\mathcal{G}_{+}$is Cesàro bounded in $L^{p}(\nu)$ we know that for almost every $x$ the function $t \mapsto H_{t}(x)$ belongs to $A_{p}^{+}$with a constant independent of $x$. Then, for each $R>0,(1.3)$ and Theorem 2.2 give

$$
\begin{aligned}
\int_{X}\left[\mathcal{T}_{\varphi_{L}, M}^{*} f(x)\right]^{p} d \nu(x) & =\frac{1}{R} \int_{0}^{R} \int_{X}\left[T^{t}\left(\mathcal{T}_{\varphi_{L}, M}^{*} f\right)(x)\right]^{p} H_{t}(x) d \nu(x) d t \\
& \leq \int_{X} \frac{1}{R} \int_{0}^{R}\left|T_{\varphi_{L}}^{*}\left(f^{x} \chi_{\left(0, L \varepsilon_{M}+R\right)}\right)(t)\right|^{p} H_{t}(x) d t d \nu(x)
\end{aligned}
$$




$$
\begin{aligned}
& \leq C\left(\int \varphi_{L}\right)^{p} \int_{X} \frac{1}{R} \int_{0}^{L \varepsilon_{M}+R}\left|f^{x}(t)\right|^{p} H_{t}(x) d t d \nu(x) \\
& =C\left(\int \varphi_{L}\right)^{p} \frac{1}{R} \int_{0}^{L \varepsilon_{M}+R} \int_{X}\left|T^{t} f(x)\right|^{p} H_{t}(x) d \nu(x) d t \\
& =C\left(\int \varphi_{L}\right)^{p} \frac{L \varepsilon_{M}+R}{R} \int_{X}|f(x)|^{p} d \nu(x) .
\end{aligned}
$$

Letting $R$ go to infinity, we obtain

$$
\int_{X}\left[\mathcal{T}_{\varphi_{L}, M}^{*} f(x)\right]^{p} d \nu(x) \leq C\left(\int \varphi_{L}\right)^{p} \int_{X}|f(x)|^{p} d \nu(x),
$$

with a constant independent of $M$ and $L$.

Let $\psi_{L}=\varphi(L) \chi_{(0, L]}+\varphi \chi_{(L, \infty)}$. By Proposition 1.3, $T_{\varphi-\varphi_{L}, M}^{*} f(x) \leq$ $4 M \mathcal{M}_{\psi_{L}}^{+} f(x) \leq 4 M\left\|\psi_{L}\right\|_{1} \mathcal{M}^{+} f(x)$. Then

$$
\int_{X}\left[T_{\varphi-\varphi_{L}, M}^{*} f(x)\right]^{p} d \nu(x) \leq C M^{p}\left\|\psi_{L}\right\|_{1}^{p} \int_{X}|f(x)|^{p} d \nu(x) .
$$

Therefore

$$
\begin{aligned}
\int_{X}\left[\mathcal{T}_{\varphi, M}^{*} f(x)\right]^{p} d \nu(x) & \leq \int_{X}\left[\mathcal{T}_{\varphi_{L}, M}^{*} f(x)\right]^{p} d \nu(x)+\int_{X}\left[T_{\varphi-\varphi_{L}, M}^{*} f(x)\right]^{p} d \nu(x) \\
& \leq C\left[\left(\int_{\varphi_{L}}\right)^{p}+C M^{p}\left\|\psi_{L}\right\|_{1}^{p}\right] \int_{X}|f(x)|^{p} d \nu(x) .
\end{aligned}
$$

Since $\left\|\psi_{L}\right\|_{1} \rightarrow 0$ as $L \rightarrow \infty$, we have

$$
\int_{X}\left[\mathcal{T}_{\varphi, M}^{*} f(x)\right]^{p} d \nu(x) \leq C\left(\int \varphi\right)^{p} \int_{X}|f(x)|^{p} d \nu(x) .
$$

Finally, letting $M$ go to $\infty$ we are done.

\section{Proofs of Theorems 1.101 .13}

5.1. Proof of Theorem 1.10. Since $\mathcal{T}_{\varphi}^{*}$ is of strong type $(p, p)$ (Theorem 1.9 it suffices to prove the a.e. convergence for $f$ in the set $D=\left\{\mathcal{A}_{\gamma}^{+} g\right.$ : $\left.g \in L^{p}(\nu), \gamma>0\right\}$, which is dense in $L^{p}(\nu)$ (see Theorem 1.7). Assume that $f \in D$, i.e., $f=\mathcal{A}_{\gamma}^{+} g$ for some $g \in L^{p}(\nu)$ and some $\gamma>0$. In this case

$$
\begin{aligned}
\left|\mathcal{A}_{\varepsilon_{k}, \varphi}^{+} f(x)-\mathcal{A}_{\varepsilon_{k-1}, \varphi}^{+} f(x)\right| \leq & \left|\mathcal{A}_{\varepsilon_{k}, \varphi}^{+}\left(\mathcal{A}_{\gamma}^{+} g\right)(x)-\mathcal{A}_{\gamma}^{+} g(x)\right| \\
& +\left|\mathcal{A}_{\gamma}^{+} g(x)-\mathcal{A}_{\varepsilon_{k-1}, \varphi}^{+}\left(\mathcal{A}_{\gamma}^{+} g\right)(x)\right| .
\end{aligned}
$$

We can deal with both terms in the same way. We only give the details for the first one. We may assume that $\int \varphi=1$. Since $\varepsilon_{k} \rightarrow 0$ as $k \rightarrow-\infty$, there 
exists $k_{0} \leq 0$ such that $\varepsilon_{k_{0}}<\gamma^{2}$ and $\varepsilon_{k_{0}+1}<1$. Therefore,

$$
\begin{aligned}
\mid \mathcal{A}_{\varepsilon_{k}, \varphi}^{+}\left(\mathcal{A}_{\gamma}^{+} g\right)(x)- & \mathcal{A}_{\gamma}^{+} g(x) \mid \\
= & \frac{1}{\gamma \varepsilon_{k}}\left|\int_{0}^{\infty} \varphi\left(t / \varepsilon_{k}\right)\left[\int_{0}^{\gamma} T^{s+t} g(x) d s-\int_{0}^{\gamma} T^{s} g(x) d s\right] d t\right| \\
\leq & \frac{1}{\gamma \varepsilon_{k}} \int_{0}^{\sqrt{\varepsilon_{k}}} \varphi\left(t / \varepsilon_{k}\right)\left|\int_{t}^{t+\gamma} T^{s} g(x) d s-\int_{0}^{\gamma} T^{s} g(x) d s\right| d t \\
& +\frac{1}{\gamma \varepsilon_{k}} \int_{\sqrt{\varepsilon_{k}}}^{\infty} \varphi\left(t / \varepsilon_{k}\right)\left|\int_{t}^{t+\gamma} T^{s} g(x) d s-\int_{0}^{\gamma} T^{s} g(x) d s\right| d t \\
= & I_{k}+I I_{k} .
\end{aligned}
$$

It will suffice to prove that

$$
\sum_{k=-\infty}^{k_{0}} I_{k}<\infty \text { and } \sum_{k=-\infty}^{k_{0}} I I_{k}<\infty
$$

for almost every $x$. We start with $I I_{k}$. We have

$$
\begin{aligned}
I I_{k}= & \frac{1}{\gamma \varepsilon_{k}} \int_{\sqrt{\varepsilon_{k}}}^{\infty} \varphi\left(t / \varepsilon_{k}\right)\left|\int_{t}^{t+\gamma} T^{s} g(x) d s-\int_{0}^{\gamma} T^{s} g(x) d s\right| d t \\
\leq & \frac{1}{\gamma \varepsilon_{k}} \int_{\sqrt{\varepsilon_{k}}}^{\infty} \varphi\left(t / \varepsilon_{k}\right)\left(\int_{t}^{t+\gamma}\left|T^{s} g(x)\right| d s\right) d t \\
& +\frac{1}{\gamma \varepsilon_{k}} \int_{\sqrt{\varepsilon_{k}}}^{\infty} \varphi\left(t / \varepsilon_{k}\right)\left(\int_{0}^{\gamma}\left|T^{s} g(x)\right| d s\right) d t=I I_{k}^{\prime}+I I_{k}^{\prime \prime} .
\end{aligned}
$$

Now

$$
\begin{aligned}
I I_{k}^{\prime \prime} & \leq \frac{\mathcal{M}^{+} g(x)}{\varepsilon_{k}} \int_{\sqrt{\varepsilon_{k}}}^{\infty} \varphi\left(t / \varepsilon_{k}\right) d t=\mathcal{M}^{+} g(x) \int_{1 / \sqrt{\varepsilon_{k}}}^{\infty} \varphi(t) d t \\
& \leq \mathcal{M}^{+} g(x) \frac{1 / \sqrt{\varepsilon_{k}}}{1 / \sqrt{\varepsilon_{k}}-1 / \sqrt{\varepsilon_{k+1}}} \int_{1 / \sqrt{\varepsilon_{k+1}}}^{1 / \sqrt{\varepsilon_{k}}} \frac{1}{s} \int_{s}^{\infty} \varphi(t) d t d s \\
& \leq C \mathcal{M}^{+} g(x) \int_{1 / \sqrt{\varepsilon_{k+1}}}^{1 / \sqrt{\varepsilon_{k}}} \frac{1}{s} \int_{s}^{\infty} \varphi(t) d t d s .
\end{aligned}
$$

Therefore, for almost every $x$, 


$$
\begin{aligned}
\sum_{k=-\infty}^{k_{0}} I I_{k}^{\prime \prime} & \leq C \mathcal{M}^{+} g(x) \sum_{k=-\infty}^{k_{0}} \int_{1 / \sqrt{\varepsilon_{k+1}}}^{1 / \sqrt{\varepsilon_{k}}} \frac{1}{s} \int_{s}^{\infty} \varphi(t) d t d s \\
& \leq C \mathcal{M}^{+} g(x) \int_{1}^{\infty} \frac{1}{s} \int_{s}^{\infty} \varphi(t) d t d s=C \mathcal{M}^{+} g(x) \int_{1}^{\infty}(\log t) \varphi(t) d t \\
& \leq C \mathcal{M}^{+} g(x) \int_{1}^{\infty} t^{1 / 2} \varphi(t) d t<\infty
\end{aligned}
$$

On the other hand, to control $I I_{k}^{\prime}$ we use Lemma 3.1. Let $p>r>1$, let $\mathcal{G}_{r}=\left\{S^{t}: t \in \mathbb{R}\right\}$ be as in that lemma and let $\widetilde{\mathcal{M}}^{+}$be the maximal operator associated to $\mathcal{G}_{r,+}=\left\{S^{t}: t>0\right\}$. By Lemma 3.1, $\widetilde{\mathcal{M}}^{+}$is bounded from $L^{p / r}(\nu)$ into $L^{p / r}(\nu)$. Consequently, $\widetilde{\mathcal{M}}^{+}\left(|g|^{r}\right)(x)<\infty$ a.e. for $g \in L^{p}(\nu)$. Applying Hölder's inequality, we have

$$
\begin{aligned}
I I_{k}^{\prime} \leq & \frac{1}{\gamma^{1 / r} \varepsilon_{k}}\left(\widetilde{\mathcal{M}}^{+}|g|^{r}(x)\right)^{1 / r} \int_{\sqrt{\varepsilon_{k}}}^{\infty}(t+\gamma)^{1 / r} \varphi\left(t / \varepsilon_{k}\right) d t \\
\leq & \frac{C}{\varepsilon_{k}}\left(\widetilde{\mathcal{M}}+\left.g\right|^{r}(x)\right)^{1 / r} \int_{\sqrt{\varepsilon_{k}}}^{\infty} \varphi\left(t / \varepsilon_{k}\right) d t \\
& +\frac{C}{\gamma^{1 / r} \varepsilon_{k}}\left(\widetilde{\mathcal{M}}^{+}|g|^{r}(x)\right)^{1 / r} \int_{\sqrt{\varepsilon_{k}}}^{\infty} t^{1 / r} \varphi\left(t / \varepsilon_{k}\right) d t .
\end{aligned}
$$

By the lacunarity of the sequence and the property of $\varphi$, we have, for a.e. $x$,

$$
\begin{aligned}
& \sum_{k=-\infty}^{k_{0}} I I_{k}^{\prime} \\
& \quad \leq C\left(\widetilde{\mathcal{M}}^{+}|g|^{r}(x)\right)^{1 / r}\left(\sum_{k=-\infty}^{k_{0}} \int_{1 / \sqrt{\varepsilon_{k}}}^{\infty} \varphi(t) d t+\sum_{k=-\infty}^{k_{0}} \frac{\varepsilon_{k}^{1 / r}}{\gamma^{1 / r}} \int_{1 / \sqrt{\varepsilon_{k}}}^{\infty} t^{1 / r} \varphi(t) d t\right) \\
& \quad \leq C\left(\widetilde{\mathcal{M}}^{+}|g|^{r}(x)\right)^{1 / r}\left(\int_{1}^{\infty}(\log t) \varphi(t) d t+\sum_{k=-\infty}^{k_{0}} \frac{\varepsilon_{k}^{1 / r}}{\gamma^{1 / r}} \int_{1}^{\infty} t^{1 / r} \varphi(t) d t\right)<\infty .
\end{aligned}
$$

So far, we have proved the second inequality in (5.1). To prove the first inequality, we notice that

$$
I_{k}=\frac{1}{\gamma \varepsilon_{k}} \int_{0}^{\sqrt{\varepsilon_{k}}} \varphi\left(t / \varepsilon_{k}\right)\left|\int_{t}^{t+\gamma} T^{s} g(x) d s-\int_{0}^{\gamma} T^{s} g(x) d s\right| d t
$$




$$
\begin{aligned}
& \leq \frac{1}{\gamma \varepsilon_{k}} \int_{0}^{\sqrt{\varepsilon_{k}}} \varphi\left(t / \varepsilon_{k}\right)\left(\int_{0}^{t}\left|T^{s} g(x)\right| d s+\int_{\gamma}^{t+\gamma}\left|T^{s} g(x)\right| d s\right) d t \\
& \leq \frac{1}{\gamma \varepsilon_{k}} \int_{0}^{\sqrt{\varepsilon_{k}}} \varphi\left(t / \varepsilon_{k}\right)\left(\int_{0}^{\sqrt{\varepsilon_{k}}}\left|T^{s} g(x)\right| d s+\int_{\gamma}^{\sqrt{\varepsilon_{k}}+\gamma}\left|T^{s} g(x)\right| d s\right) d t \\
& =I_{k}^{\prime}+I_{k}^{\prime \prime} .
\end{aligned}
$$

Now

$$
\begin{aligned}
I_{k}^{\prime} & \leq \frac{\sqrt{\varepsilon_{k}}}{\gamma \varepsilon_{k}} \mathcal{M}^{+} g(x) \int_{0}^{\sqrt{\varepsilon_{k}}} \varphi\left(t / \varepsilon_{k}\right) d t \\
& \leq \frac{\sqrt{\varepsilon_{k}}}{\gamma} \mathcal{M}^{+} g(x) \int_{0}^{\infty} \varphi(t) d t=\frac{\sqrt{\varepsilon_{k}}}{\gamma} \mathcal{M}^{+} g(x),
\end{aligned}
$$

so that $\sum_{k=-\infty}^{k_{0}} I_{k}^{\prime} \leq \gamma^{-1}\left(\sum_{k=-\infty}^{k_{0}} \sqrt{\varepsilon_{k}}\right) \mathcal{M}^{+} g(x)<\infty$ a.e.

To control $I_{k}^{\prime \prime}$ we use again Lemma 3.1 . Let $p>r>1$, let $\Gamma_{\gamma}=\left\{S^{t}\right.$ : $t \in \mathbb{R}\}$ be as in that lemma and let $\widetilde{\mathcal{M}}^{+}$be the maximal operator associated to $\mathcal{G}_{r,+}=\left\{S^{t}: t>0\right\}$. By Lemma 3.1, $\widetilde{\mathcal{M}}^{+}$is bounded from $L^{p / r}(\nu)$ into $L^{p / r}(\nu)$. Consequently, $\widetilde{\mathcal{M}}^{+}\left(|g|^{r}\right)(x)<\infty$ a.e. for $g \in L^{p}(\nu)$. Applying Hölder's inequality, we have

$$
\begin{aligned}
I_{k}^{\prime \prime} & =\frac{1}{\gamma}\left(\int_{0}^{1 / \sqrt{\varepsilon_{k}}} \varphi(t) d t\right)\left(\int_{\gamma}^{\sqrt{\varepsilon_{k}}+\gamma}\left|T^{s} g(x)\right| d s\right) \\
& \leq \frac{1}{\gamma}\left(\int_{0}^{1 / \sqrt{\varepsilon_{k}}} \varphi(t) d t\right)\left(\int_{\gamma}^{\sqrt{\varepsilon_{k}}+\gamma}\left|T^{s} g(x)\right|^{r} d s\right)^{1 / r}\left(\sqrt{\varepsilon_{k}}\right)^{1 / r^{\prime}} \\
& \leq \frac{1}{\gamma}\left(\int_{0}^{1 / \sqrt{\varepsilon_{k}}} \varphi(t) d t\right)\left(\sqrt{\varepsilon_{k}}+\gamma\right)^{1 / r}\left(\sqrt{\varepsilon_{k}}\right)^{1 / r^{\prime}}\left(\widetilde{\mathcal{M}}^{+}\left(|g|^{r}\right)(x)\right)^{1 / r} \\
& \leq \frac{(2 \gamma)^{1 / r}}{\gamma}\left(\int_{0}^{1 / \sqrt{\varepsilon_{k}}} \varphi(t) d t\right)\left(\sqrt{\varepsilon_{k}}\right)^{1 / r^{\prime}}\left(\widetilde{\mathcal{M}}+\left(|g|^{r}\right)(x)\right)^{1 / r} \\
& \leq \frac{2^{1 / r}}{\gamma^{1 / r^{\prime}}}\left(\int_{0}^{\infty} \varphi(t) d t\right)\left(\sqrt{\varepsilon_{k}}\right)^{1 / r^{\prime}}\left(\widetilde{\mathcal{M}^{+}}\left(|g|^{r}\right)(x)\right)^{1 / r} .
\end{aligned}
$$

Therefore $\sum_{k=-\infty}^{k_{0}} I_{k}^{\prime \prime}<\infty$ a.e. This finishes the proof of 5.1 .

5.2. Proof of Theorem 1.11. It suffices to prove the a.e. convergence in the case $p>1$. The other statements follow from the results already proved and standard arguments. We also notice that it is enough to prove the a.e. convergence for $f$ in the set $\widetilde{D}=\left\{\mathcal{A}_{\varepsilon}^{+} g: g \in L^{p}(\nu) \cap L^{\infty}(\nu), \varepsilon>0\right\}$, 
which is dense in $L^{p}(\nu)$ (see Theorem 1.7). So, we take $f=\mathcal{A}_{\varepsilon}^{+} g, g \in$ $L^{p}(\nu) \cap L^{\infty}(\nu)$, and we follow the proof of Theorem 1.10 except for the estimates for $I I_{k}^{\prime}$ (see $(5.2)$ ), which is now estimated in the following way:

$$
I I_{k}^{\prime}=\frac{1}{\gamma \varepsilon_{k}} \int_{\sqrt{\varepsilon_{k}}}^{\infty} \varphi\left(t / \varepsilon_{k}\right)\left(\int_{t}^{t+\gamma}\left|T^{s} g(x)\right| d s\right) d t \leq \frac{\|g\|_{\infty}}{\varepsilon_{k}} \int_{\sqrt{\varepsilon_{k}}}^{\infty} \varphi\left(t / \varepsilon_{k}\right) d t .
$$

The conclusion follows with the same computations as in (5.3) and (5.4).

5.3. Proof of Theorem 1.12, As before, it suffices to prove the a.e. convergence in the case $p>1$. To prove the convergence of $\mathcal{T}_{N}^{2} f(x)$, it is enough to establish it for $f \in A \oplus \widetilde{B}$, where $A=\left\{f \in L^{p}(\nu): f\left(\tau_{t} x\right)=\right.$ $f(x)$ for all $t>0\}$ and $\widetilde{B}$ is the linear manifold generated by

$$
\left\{f(x)-\mathcal{A}_{\gamma}^{+} f(x): f \in L^{p}(\nu) \cap L^{\infty}(\nu), \gamma>0\right\},
$$

since it follows from Theorem 3.3 that $A \oplus \widetilde{B}$ is dense in $L^{p}(\nu)$. If $f \in A$ there is nothing to prove. Suppose $f=g-\mathcal{A}_{\gamma}^{+} g, g \in L^{p}(\nu) \cap L^{\infty}(\nu), \gamma>0$. Then

$$
\left|\mathcal{A}_{\varepsilon_{k}, \varphi}^{+} f(x)-\mathcal{A}_{\varepsilon_{k-1}, \varphi}^{+} f(x)\right| \leq\left|\mathcal{A}_{\varepsilon_{k}, \varphi}^{+}\left(g-\mathcal{A}_{\gamma}^{+} g\right)(x)\right|+\left|\mathcal{A}_{\varepsilon_{k-1}, \varphi}^{+}\left(g-\mathcal{A}_{\gamma}^{+} g\right)(x)\right| .
$$

Again, we can deal with both terms in the same way. Since $\varepsilon_{k} \rightarrow \infty$ as $k \rightarrow \infty$, there exists $k_{0}$ such that for all $k \geq k_{0}-1$ we have $\varepsilon_{k}>\gamma$ and $\varepsilon_{k}>1$. Therefore, for $k \geq k_{0}$,

$$
\begin{aligned}
\mid \mathcal{A}_{\varepsilon_{k}, \varphi}^{+}\left(g-\mathcal{A}_{\gamma}^{+} g\right) & (x) \mid \\
= & \left|\int_{0}^{\infty} T^{t} g(x) \varphi_{\varepsilon_{k}}(t) d t-\frac{1}{\gamma} \int_{0}^{\gamma} \int_{0}^{\infty} T^{t+s} g(x) \varphi_{\varepsilon_{k}}(t) d t d s\right| \\
= & \left|\int_{0}^{\infty} T^{t} g(x) \varphi_{\varepsilon_{k}}(t) d t-\frac{1}{\gamma} \int_{0}^{\gamma} \int_{s}^{\infty} T^{t} g(x) \varphi_{\varepsilon_{k}}(t-s) d t d s\right| \\
\leq & \frac{1}{\gamma} \int_{0}^{\gamma} \frac{1}{\varepsilon_{k}} \int_{0}^{s}\left|T^{t} g(x)\right| \varphi\left(t / \varepsilon_{k}\right) d t d s \\
& +\frac{1}{\gamma} \int_{0}^{\gamma} \frac{1}{\varepsilon_{k}} \int_{s}^{\infty}\left|T^{t} g(x)\right|\left|\varphi\left(t / \varepsilon_{k}\right)-\varphi\left(t / \varepsilon_{k}-s / \varepsilon_{k}\right)\right| d t d s .
\end{aligned}
$$

Notice that, by the hypothesis on the function $\varphi$,

$$
\int_{s}^{\infty}\left|\varphi\left(t / \varepsilon_{k}\right)-\varphi\left((t-s) / \varepsilon_{k}\right)\right| d t=\int_{s}^{\infty}\left[\varphi\left((t-s) / \varepsilon_{k}\right)-\varphi\left(t / \varepsilon_{k}\right)\right] d t=\int_{0}^{s} \varphi\left(t / \varepsilon_{k}\right) d t .
$$


Using that $\left\|T^{t} g\right\|_{\infty} \leq\|g\|_{\infty}$, we have

$$
\left|\mathcal{A}_{\varepsilon_{k}, \varphi}^{+}\left(g-\mathcal{A}_{\gamma}^{+} g\right)(x)\right| \leq \frac{2}{\gamma}\|g\|_{\infty} \int_{0}^{\gamma} \frac{1}{\varepsilon_{k}} \int_{0}^{s} \varphi\left(t / \varepsilon_{k}\right) d t d s .
$$

For $s<\gamma$,

$$
\begin{aligned}
\frac{1}{\varepsilon_{k}} \int_{0}^{s} \varphi\left(t / \varepsilon_{k}\right) d t & =\int_{0}^{s / \varepsilon_{k}} \varphi(t) d t \leq \frac{1 / \varepsilon_{k-1}}{1 / \varepsilon_{k-1}-1 / \varepsilon_{k}} \int_{\gamma / \varepsilon_{k}}^{\gamma / \varepsilon_{k-1}} \frac{1}{u} \int_{0}^{u} \varphi(t) d t d u \\
& \leq C \int_{\gamma / \varepsilon_{k}}^{\gamma / \varepsilon_{k-1}} \frac{1}{u} \int_{0}^{u} \varphi(t) d t d u .
\end{aligned}
$$

Then

$$
\begin{aligned}
& \sum_{k=k_{0}}^{\infty} \mid \mathcal{A}_{\varepsilon_{k}, \varphi}^{+}(g-\left.\mathcal{A}_{\gamma}^{+} g\right)(x) \mid \leq C\|g\|_{\infty} \sum_{k=k_{0}}^{\infty} \int_{\gamma / \varepsilon_{k}}^{\gamma / \varepsilon_{k-1}} \frac{1}{s} \int_{0}^{s} \varphi(t) d t d s \\
& \leq C\|g\|_{\infty} \int_{0}^{1} \frac{1}{s} \int_{0}^{s} \varphi(t) d t d s=C\|g\|_{\infty} \int_{0}^{1}|\log t| \varphi(t) d t<\infty,
\end{aligned}
$$

and we are done.

5.4. Proof of Theorem 1.13. It suffices to prove the a.e. convergence. To prove the a.e. convergence of $\mathcal{T}_{N}^{2} f(x)$, it is enough to establish it for $f \in A \oplus B$, where $A$ and $B$ are the sets in the proof of Theorem 3.3 , that is, $A=\left\{f \in L^{p}(\nu): f\left(\tau_{t} x\right)=f(x)\right.$ for all $\left.t>0\right\}$ and $B$ is the linear manifold generated by $\left\{f(x)-\mathcal{A}_{\gamma}^{+} f(x): f \in L^{p}(\nu), \gamma>0\right\}$. If $f \in A$ there is nothing to prove. Suppose $f=g-\mathcal{A}_{\gamma}^{+} g, g \in L^{p}(\nu), \gamma>0$. Then

$$
\left|\mathcal{A}_{\varepsilon_{k}}^{+} f(x)-\mathcal{A}_{\varepsilon_{k-1}}^{+} f(x)\right| \leq\left|\mathcal{A}_{\varepsilon_{k}}^{+}\left(g-\mathcal{A}_{\gamma}^{+} g\right)(x)\right|+\left|\mathcal{A}_{\varepsilon_{k-1}}^{+}\left(g-\mathcal{A}_{\gamma}^{+} g\right)(x)\right| .
$$

Again, we can deal with both terms in the same way. Since $\varepsilon_{k} \rightarrow \infty$ as $k \rightarrow \infty$, there exists $k_{0}$ such that for all $k \geq k_{0}-1$ we have $\varepsilon_{k}>\gamma$. Therefore, for $k \geq k_{0}$, using (3.16),

$$
\begin{aligned}
\left|\mathcal{A}_{\varepsilon_{k}}^{+}\left(g-\mathcal{A}_{\gamma}^{+} g\right)(x)\right| & \leq \frac{1}{\varepsilon_{k}} \int_{0}^{\gamma} T^{t}|g|(x) d t+\frac{1}{\varepsilon_{k}} \int_{\varepsilon_{k}}^{\gamma+\varepsilon_{k}} T^{t}|g|(x) d t \\
& =I_{k}+I I_{k} .
\end{aligned}
$$

It is clear that $\sum_{k>k_{0}} I_{k} \leq \sum_{k>k_{0}}\left(\gamma / \varepsilon_{k}\right) \mathcal{M}^{+} g(x)<\infty$ a.e. since $g \in L^{p}(\nu)$. To control the other term we use again Lemma 3.1. Let $p>r>1$, let $\mathcal{G}_{r}=\left\{S^{t}: t \in \mathbb{R}\right\}$ be as in that lemma and let $\widetilde{\mathcal{M}}^{+}$be the maximal operator associated to $\mathcal{G}_{r,+}=\left\{S^{t}: t>0\right\}$. By Lemma 3.1, $\widetilde{\mathcal{M}}^{+}$is bounded from $L^{p / r}(\mu)$ into $L^{p / r}(\mu)$. Consequently, $\widetilde{\mathcal{M}}^{+}\left(|g|^{r}\right)(x)<\infty$ a.e. for $g \in L^{p}(\mu)$. It 
follows that

$$
\begin{aligned}
I I_{k} & \leq \frac{1}{\varepsilon_{k}}\left(\int_{\varepsilon_{k}}^{\gamma+\varepsilon_{k}}\left(T^{t}|g|(x)\right)^{r} d t\right)^{1 / r} \gamma^{1 / r^{\prime}} \\
& \leq \frac{\left(\gamma+\varepsilon_{k}\right)^{1 / r}}{\varepsilon_{k}}\left(\frac{1}{\gamma+\varepsilon_{k}} \int_{0}^{\gamma+\varepsilon_{k}} S^{t}\left(|g|^{r}\right)(x) d t\right)^{1 / r} \gamma^{1 / r^{\prime}} \\
& \leq \frac{\left(\gamma+\varepsilon_{k}\right)^{1 / r} \gamma^{1 / r^{\prime}}}{\varepsilon_{k}}\left[\widetilde{\mathcal{M}}^{+}\left(|g|^{r}\right)(x)\right]^{1 / r} \leq \frac{2^{1 / r} \gamma^{1 / r^{\prime}}}{\varepsilon_{k}^{1 / r^{\prime}}}\left[\widetilde{\mathcal{M}}^{+}\left(|g|^{r}\right)(x)\right]^{1 / r} .
\end{aligned}
$$

Therefore

$$
\sum_{k \geq k_{0}} I I_{k} \leq \sum_{k \geq k_{0}} \frac{2^{1 / r} \gamma^{1 / r^{\prime}}}{\varepsilon_{k}^{1 / r^{\prime}}}\left[\widetilde{\mathcal{M}}^{+}\left(|g|^{r}\right)(x)\right]^{1 / r}<\infty \quad \text { a.e. }
$$

Consequently,

$$
\sum_{k \geq k_{0}}\left|\mathcal{A}_{\varepsilon_{k}, \varphi}^{+}\left(g-\mathcal{A}_{\gamma}^{+} g\right)(x)\right| \leq \sum_{k \geq k_{0}}\left(I_{k}+I I_{k}\right)<\infty \quad \text { a.e., }
$$

as we wished to prove.

6. Examples of Cesàro bounded semigroups. The aim of this section is to provide more examples of Cesàro bounded semigroups. We follow the arguments in [18]. Given a nonsingular flow $\Gamma=\left\{\tau_{t}: t \in \mathbb{R}\right\}$, we first study the groups $T^{t} f(x)=f\left(\tau_{t} x\right)$ and then the general groups $T^{t} f(x)=g_{t}(x) f\left(\tau_{t} x\right)$. We frequently use Remark 3.5 .

6.1. The group $T^{t} f(x)=f\left(\tau_{t} x\right)$. We start by giving examples in the basic setting of the interval $[0,1)$.

EXAmple 6.1 . Let $X=[0,1)$ with the Lebesgue $\sigma$-algebra. Let $d \nu=$ $w(x) d x$, where $w(x)=x^{\beta}$. Consider the flow $\tau_{t} x=x+t(\bmod 1)$ and the group $T^{t} f(x)=f\left(\tau_{t} x\right)$. In this case $H_{t}(x)=w\left(\tau_{t} x\right) / w(x)$. In Example 1.6 we have seen that if $-1<\beta \leq 0$ then the function $t \mapsto H_{t}(x)$ belongs to $A_{1}^{+}$ with a constant independent of $x$. Therefore, by Remark 3.5, $\mathcal{G}_{+}$is Cesàro bounded in $L^{1}(\nu)$. We already know (see Example 1.6) that the semigroup $\mathcal{G}_{-}=\left\{T^{t}: t<0\right\}$ is not Cesàro bounded in $L^{1}(\nu)$ for $\beta<0$.

In the same way, if $w(x)=(1-x)^{\beta}$ and $-1<\beta \leq 0$ then the function $t \mapsto H_{t}(x)$ belongs to $A_{1}^{-}$with a constant independent of $x$. It follows from the theory of one-sided weights (see Remarks 2.1) that if $0 \leq \beta<p-1, p>1$, and $w(x)=(1-x)^{\beta}$ or $w(x)=x^{\beta},-1<\beta \leq 0$, then $t \mapsto H_{t}(x)$ belongs to $A_{p}^{+}$with a constant independent of $x$. Therefore, in those cases, the semigroup $\mathcal{G}_{+}$is Cesàro bounded in $L^{p}(\nu)$. As in Example 1.6, the semigroup $\mathcal{G}_{-}=\left\{T^{t}: t<0\right\}$ is not Cesàro bounded in $L^{p}(\nu)$ for $\beta \neq 0$. 
We can see that in the above example the flow preserves a measure $\mu$ (the Lebesgue measure) which is equivalent to $\nu$ (that is, $\nu(E)=0$ if and only if $\mu(E)=0)$. One may ask if this is always the case when we consider Cesàro bounded semigroups of the form $T^{t} f(x)=f\left(\tau_{t} x\right)$. The answer is affirmative when the measure $\nu$ is finite. We state it as a theorem.

TheOREM 6.2. Let $(X, \mathcal{F}, \nu)$ be a finite measure space and let $\Gamma=\left\{\tau_{t}\right.$ : $t \in \mathbb{R}\}$ be a nonsingular flow on $X$. Let $\mathcal{G}=\left\{T^{t}: t \in \mathbb{R}\right\}$ be the group defined as $T^{t} f(x)=f\left(\tau_{t} x\right)$. Let $1 \leq p<\infty$. If the semigroup $\mathcal{G}_{+}$is Cesàro bounded in $L^{p}(\nu)$ then there exists a finite measure $\mu$ preserved by the flow and equivalent to $\nu$.

The proof is as in [18, proof of Theorem 1, p. 545]. Therefore, we do not include it.

\subsection{Nontrivial examples of Cesàro bounded general semi-} groups. Consider a $\sigma$-finite measure space $(X, \mathcal{F}, \nu)$ and a nonsingular flow $\left\{\tau_{t}: t \in \mathbb{R}\right\}$ on $X$. Recall that the transformation $\tau_{t}$ is ergodic if $\tau_{-t}(E)=E$ for a measurable set $E$ implies that $\nu(E)=0$ or $\nu(X \backslash E)=0$.

Let $\mathcal{G}=\left\{T^{t}: t \in \mathbb{R}\right\}$ be the group defined by $T^{t} f(x)=\left(\overline{J_{t}}(x)\right)^{1 / p} f\left(\tau_{t} x\right)$, $1 \leq p<\infty$. Clearly, each $T^{t}$ is an isometry on $L^{p}(\nu)$. Therefore $\mathcal{G}^{+}=$ $\left\{T^{t}: t>0\right\}$ is Cesàro bounded in $L^{p}(\nu)$. Our next result yields nontrivial examples of Cesàro bounded semigroups, in the sense that the operators $T^{t}$, $t>0$, are not isometries, moreover they are not uniformly bounded.

TheOREM 6.3. Let $(X, \mathcal{F}, \nu)$ be a nonatomic finite measure space and let $\Gamma=\left\{\tau_{t}: t \in \mathbb{R}\right\}$ be a nonsingular flow on $X$. Assume that $\tau_{t}$ is ergodic for some $t$ with respect to $\nu$. Let $1 \leq p<\infty$. Then there exists a group of positive operators $\mathcal{S}_{p}=\left\{T^{t}: t \in \mathbb{R}\right\}$ induced by the flow, acting on measurable functions, such that

(1) the semigroup $\mathcal{S}_{p}^{+}=\left\{T^{t}: t>0\right\}$ is Cesàro bounded in $L^{p}(\nu)$,

(2) the semigroup $\mathcal{S}_{p}^{+}$is not uniformly bounded in $L^{p}(\nu)$, that is, there is no $C>0$ such that $\int_{X}\left|T^{t} f\right|^{p} d \nu \leq C \int_{X}|f|^{p} d \nu$ for all $t>0$ and all $f \in L^{p}(\nu)$.

Notice that the result is a generalization of Theorem 7 in [18].

Proof. We start by proving the case $p=1$. We do it in two steps. We again use ideas of Rubio de Francia.

1) Let $p=1$ and assume that there exists a finite measure $\mu$ equivalent to $\nu$ and preserved by the flow $\Gamma$. Let $\mathcal{M}_{\mu}^{-}$be the maximal operator defined by $\mathcal{M}_{\mu}^{-} f(x)=\sup _{\varepsilon>0} \varepsilon^{-1} \int_{-\varepsilon}^{0}\left|f\left(\tau_{t} x\right)\right| d t$. We know that $\mathcal{M}_{\mu}^{-}$is bounded on $L^{2}(\mu)$, i.e., there exists a constant $A>0$ such that $\left\|\mathcal{M}_{\mu}^{-} f\right\|_{2, \mu} \leq A\|f\|_{2, \mu}$. Let $f>0, f \in L^{2}(\mu) \backslash L^{\infty}(\mu)$. Let $w=\sum_{i=0}^{\infty} A^{-i}\left(\mathcal{M}_{\mu}^{-}\right)^{(i)} f$, where $\left(\mathcal{M}_{\mu}^{-}\right)^{(i)}$ is the $i$ th iteration of $\mathcal{M}_{\mu}^{-}$. Then $w \geq f>0, w \in L^{2}(\mu), w$ is finite a.e., 
$w \notin L^{\infty}(\mu)$ and $\mathcal{M}_{\mu}^{-} w \leq \sum_{i=0}^{\infty} A^{-i}\left(\mathcal{M}_{\mu}^{-}\right)^{(i+1)} w \leq A w$ a.e., which implies that

for a.e. $x$ the functions $t \mapsto w\left(\tau_{t} x\right)$

belong to $A_{1}^{+}$with a uniform constant.

Let $u=d \mu / d \nu$ be the Radon-Nikodym derivative. Let

$$
g_{t}(x)=\frac{u(x) w(x)}{\left(u\left(\tau_{t} x\right) w\left(\tau_{t} x\right)\right)}
$$

and let $\mathcal{S}_{1}=\left\{T^{t}: t \in \mathbb{R}\right\}$ be the group defined as $T^{t} f(x)=g_{t}(x) f\left(\tau_{t} x\right)$. Since $J_{t}(x)=u(x) / u\left(\tau_{t} x\right)$, it follows from (6.1) that the semigroup $\mathcal{S}_{1}=$ $\left\{T^{t}: t>0\right\}$ is Cesàro bounded in $L^{1}(\nu)$ (this can be seen directly or by applying Remark 3.5).

Now assume towards a contradiction that the semigroup $\mathcal{S}_{1}$ is uniformly bounded in $L^{1}(d \nu)$. It is clear that then there exists a constant $C>0$ such that

$$
\int_{X} \frac{u(x) w(x)}{u\left(\tau_{t} x\right) w\left(\tau_{t} x\right)} f\left(\tau_{t} x\right) d \nu \leq C \int_{X} f(x) d \nu=\int_{X} f\left(\tau_{t} x\right) \frac{u(x)}{u\left(\tau_{t} x\right)} d \nu
$$

for all $f \geq 0$. This inequality implies, for all $t>0, w(x) \leq C w\left(\tau_{t} x\right)$ a.e. $x$, which implies that $w \in L^{\infty}(d \nu)$, by applying the ergodicity of some $\tau_{t}$, which is a contradiction.

2) Let $p=1$ and assume that there is no finite measure $\mu$ equivalent to $\nu$ and preserved by the flow $\Gamma$. Let $1<q<\infty$ and let $q^{\prime}$ be the conjugate exponent. We consider the group $\mathcal{G}_{q^{\prime}}=\left\{T^{t}: t \in \mathbb{R}\right\}$ where $T^{t} f(x)=\left(\overline{J_{t}}(x)\right)^{1 / q^{\prime}} f\left(\tau_{t} x\right)$. Since the operators $T^{t}$ are positive isometries on $L^{q^{\prime}}(\nu)$, the maximal operator $\mathcal{M}_{q^{\prime}}^{-} f(x)=\sup _{\varepsilon>0} \varepsilon^{-1} \int_{-\varepsilon}^{0}\left|T^{t} f(x)\right| d t$ is bounded on $L^{q^{\prime}}(\nu)$, i.e., there exists $A>0$ such that $\left\|\mathcal{M}_{q^{\prime}}^{-} f\right\|_{q^{\prime}, \nu} \leq A\|f\|_{q^{\prime}, \nu}$. Let $f>0, f \in L^{q^{\prime}}(\nu)$. Let $w=\sum_{i=0}^{\infty} A^{-i}\left(\mathcal{M}_{q^{\prime}}^{-}\right)^{(i)} f$, where $\left(\mathcal{M}_{q^{\prime}}^{-}\right)^{(i)}$ is the $i$ th iteration of $\mathcal{M}_{q^{\prime}}^{-}$. Then $w \geq f>0, w \in L^{q^{\prime}}(\nu), w$ is finite a.e. and $\mathcal{M}_{q^{\prime}}^{-} w \leq \sum_{i=0}^{\infty} A^{-i}\left(\mathcal{M}_{q^{\prime}}^{-}\right)^{(i+1)} w \leq A w$ a.e., which means that

for a.e. $x$ the functions $t \mapsto\left(J_{t}(x)\right)^{1 / q^{\prime}} w\left(\tau_{t} x\right)$

belong to $A_{1}^{+}$with a uniform constant.

Let $\overline{g_{t}}(x)=\frac{w(x)}{w\left(\tau_{t} x\right)}\left(\overline{J_{t}}(x)\right)^{1 / q}$ and let $\mathcal{S}_{1}=\left\{T^{t}: t \in \mathbb{R}\right\}$ be the group defined as $T^{t} f(x)=\overline{g_{t}}(x) f\left(\tau_{t} x\right)$. As before, it follows from $(6.2)$ that the semigroup $\mathcal{S}_{1}=\left\{T^{t}: t>0\right\}$ is Cesàro bounded in $L^{1}(\nu)$ (this can be seen directly or by applying Remark 3.5.

Now assume towards a contradiction that the semigroup $\mathcal{S}_{1}$ is uniformly bounded in $L^{1}(d \nu)$. It is clear that then there exists a constant $C>0$ such 
that

$$
\int_{X} \frac{w(x)}{w\left(\tau_{t} x\right)}\left(\overline{J_{t}}(x)\right)^{1 / q} f\left(\tau_{t} x\right) d \nu \leq C \int_{X} f(x) d \nu=\int_{X} f\left(\tau_{t} x\right) \overline{J_{t}}(x) d \nu
$$

for all $f \geq 0$. This inequality implies, for all $t>0$,

$$
w(x) \leq C w\left(\tau_{t} x\right)\left(\overline{J_{t}}(x)\right)^{1 / q^{\prime}} \text { a.e. } x .
$$

Raising to the power $q^{\prime}$, multiplying by $\chi_{A}\left(\tau_{t} x\right)$, where $A$ is any measurable set, and integrating on $X$, we have

$$
\int_{X} \chi_{A}\left(\tau_{t} x\right) w^{q^{\prime}}(x) d \nu=\int_{\tau_{-t} A} w^{q^{\prime}} d \nu \leq C \int_{A} w^{q^{\prime}} d \nu=\int_{X} \chi_{A}(x) w^{q^{\prime}}(x) d \nu
$$

for all $t>0$. This condition implies that if $\widetilde{T}^{t} f(x)=f\left(\tau_{t} x\right)$ and $d \widetilde{\nu}=w^{q^{\prime}} d \nu$ then the semigroup $\left\{\widetilde{T}^{t}: t>0\right\}$ is Cesàro bounded in $L^{1}(d \widetilde{\nu})$. By Theorem 6.2 , there exists a finite measure $\mu$ preserved by the flow and equivalent to $\widetilde{\nu}$, and hence to $\nu$. That is a contradiction.

3) Let $p>1$ and assume that there exists a finite measure $\mu$ equivalent to $\nu$ and preserved by the flow $\Gamma$. As in the case $p=1$ but with the maximal operator $\mathcal{M}_{\mu}^{+} f(x)=\sup _{\varepsilon>0} \varepsilon^{-1} \int_{0}^{\varepsilon}\left|f\left(\tau_{t} x\right)\right| d t$, there exists $w \in L^{2}(\mu) \backslash L^{\infty}(\mu)$ such that $\mathcal{M}_{\mu}^{+} w \leq A w$ a.e., which means that for a.e. $x$ the functions $t \mapsto$ $w\left(\tau_{t} x\right)$ belong to $A_{1}^{-}$with a uniform constant. Let $v=w^{1-p}$. Then it is well known (see Remarks 2.1) that

for a.e. $x$ the functions $t \mapsto v\left(\tau_{t} x\right)$

belong to $A_{p}^{+}$with a uniform constant.

Let $u=d \mu / d \nu$ be the Radon-Nikodym derivative. Let

$$
g_{t}(x)=\left(\frac{u(x) v(x)}{u\left(\tau_{t} x\right) v\left(\tau_{t} x\right)}\right)^{1 / p}
$$

and let $\mathcal{S}_{p}=\left\{T^{t}: t \in \mathbb{R}\right\}$ be the group defined as $T^{t} f(x)=g_{t}(x) f\left(\tau_{t} x\right)$. It follows from (6.3) that the semigroup $\mathcal{S}_{p}=\left\{T^{t}: t>0\right\}$ is Cesàro bounded in $L^{p}(\nu)$ (this can be seen by applying Remark 3.5).

Now assume that the semigroup $\mathcal{S}_{p}$ is uniformly bounded in $L^{p}(d \nu)$. It is clear that then there exists a constant $C>0$ such that

$$
\int_{X} \frac{u(x) v(x)}{u\left(\tau_{t} x\right) v\left(\tau_{t} x\right)}\left|f\left(\tau_{t} x\right)\right|^{p} d \nu \leq C \int_{X}|f(x)|^{p} d \nu=\int_{X}\left|f\left(\tau_{t} x\right)\right|^{p} \frac{u(x)}{u\left(\tau_{t} x\right)} d \nu
$$

for all $f \geq 0$. As in the case $p=1$, this implies that $w \in L^{\infty}(d \nu)$, a contradiction.

4) Let $p>1$ and assume that there is no finite measure $\mu$ equivalent to $\nu$ and preserved by the flow $\Gamma$. As in case 2), using $\mathcal{M}_{q^{\prime}}^{-} f(x)=$ $\sup _{\varepsilon>0} \varepsilon^{-1} \int_{-\varepsilon}^{0}\left|T^{t} f(x)\right| d t$, we have a function $w \in L^{q^{\prime}}(\nu)$ such that $\mathcal{M}_{q^{\prime}}^{-} w \leq$ 
$A w$ a.e., which means that for a.e. $x$ the functions $t \mapsto\left(J_{t}(x)\right)^{1 / q^{\prime}} w\left(\tau_{t} x\right)$ belong to $A_{1}^{-}$with a uniform constant. Then

for a.e. $x$ the functions $t \mapsto\left(\left(J_{t}(x)\right)^{1 / q^{\prime}} w\left(\tau_{t} x\right)\right)^{1-p}$ belong to $A_{p}^{+}$with a uniform constant.

Let

$$
\overline{g_{t}}(x)=\left(\frac{w(x)}{w\left(\tau_{t} x\right)}\right)^{\frac{1-p}{p}}\left(\overline{J_{t}}(x)\right)^{\frac{1}{p}+\frac{1}{p^{\prime} q^{\prime}}}
$$

and let $\mathcal{S}_{p}=\left\{T^{t}: t \in \mathbb{R}\right\}$, where $T^{t} f(x)=\overline{g_{t}}(x) f\left(\tau_{t} x\right)$. As before, it follows from 6.4 that the semigroup $\mathcal{S}_{p}=\left\{T^{t}: t>0\right\}$ is Cesàro bounded in $L^{p}(\nu)$.

Now we see that that the semigroup $\mathcal{S}_{p}$ is not uniformly bounded in $L^{p}(d \nu)$. Proceeding as in case 2$)$, we obtain, for all $t>0, w\left(\tau_{t} x\right)\left(\overline{J_{t}}(x)\right)^{1 / q^{\prime}} \leq$ $C w(x)$ a.e. $x$ and, for any measurable set $A$,

$$
\int_{X} \chi_{A}\left(\tau_{-t} x\right) w^{q^{\prime}}(x) d \nu \leq C \int_{X} \chi_{A}(x) w^{q^{\prime}}(x) d \nu
$$

for all $t>0$. This implies that if $\widetilde{T}^{t} f(x)=f\left(\tau_{-t} x\right)$ and $d \widetilde{\nu}=w^{q^{\prime}} d \nu$ then the semigroup $\left\{\widetilde{T}^{t}: t>0\right\}$ is Cesàro bounded in $L^{1}(d \widetilde{\nu})$. By Theorem 6.2, there exists a finite measure $\mu$ equivalent to $\widetilde{\nu}$ (and hence to $\nu$ ) and preserved by the flow. That is a contradiction.

Acknowledgments. This research has been partially supported by CAI+D-UNL, CONICET (Argentina), Spanish Government, Ministerio de Ciencia y Tecnología grants MTM2005-8350-C03-02 and MTM2008-06621C02-02, and Junta de Andalucía grants FQM-354 and FQM-01509.

\section{References}

[1] M. A. Akcoglu, R. L. Jones and P. O. Schwartz, Variation in probability, ergodic theory and analysis, Illinois J. Math. 42 (1998), 1, 154-177.

[2] E. Berkson and T. A. Gillespie, A Tauberian theorem for ergodic averages, spectral decomposability, and the dominated ergodic estimate for positive invertible operators, Positivity 7 (2003), 161-175.

[3] A. L. Bernardis, M. Lorente, F. J. Martín-Reyes, M. T. Martínez, A. de la Torre and J. L. Torrea, Differential transforms in weighted spaces, J. Fourier Anal. Appl. 12 (2006), 83-103.

[4] - , 一, - - - - , Differences of ergodic averages for Cesàro bounded operators, Quart. J. Math. 58 (2007), 137-150.

[5] M. Broise, Y. Déniel and Y. Derriennic, Maximal inequalities and ergodic theorems for Cesàro- $\alpha$ or weighted averages, in: Almost Everywhere Convergence, II (Evanston, IL, 1989), Academic Press, Boston, MA, 1991, 93-107.

[6] N. Dunford and J. T. Schwartz, Linear Operators. I, Pure Appl. Math. 7, Interscience, New York, 1958. 
[7] J. Duoandikoetxea, Fourier Analysis, Grad. Stud. Math. 29, Amer. Math. Soc., Providence, RI, 2001.

[8] D. A. Edwards, A maximal ergodic theorem for Abel means of continuous-parameter operator semigroups, J. Funct. Anal. 7 (1971), 61-70.

[9] R. Émilion, Mean-bounded operators and mean ergodic theorems, ibid. 61 (1985), $1-14$.

[10] J. García-Cuerva and J. L. Rubio de Francia, Weighted Norm Inequalities and Related Topics, North-Holland Math. Stud. 116, North-Holland, Amsterdam, 1985.

[11] T. A. Gillespie and J. L. Torrea, Weighted ergodic theory and dimension free estimates, Quart. J. Math. 54 (2003), 257-280.

[12] L. Grafakos, Classical and Modern Fourier Analysis, Pearson Education, Inc., Upper Saddle River, NJ, 2004.

[13] B. Johnson, Unconditional convergence of differences in ergodic theory, Ph.D. Dissertation, The Ohio State Univ., 1997.

[14] R. L. Jones and J. Rosenblatt, Differential and ergodic transforms, Math. Ann. 323 (2002), 525-546.

[15] U. Krengel, A necessary and sufficient condition for the validity of the local ergodic theorem, in: Probability and Information Theory (Hamilton, Ont., 1968), Springer, Berlin, 1969, 170-177.

[16] —, Ergodic Theorems, de Gruyter Stud. Math. 6, de Gruyter, Berlin, 1985.

[17] Y.-C. Li, S.-Y. Shaw and R. Sato, Boundedness and growth orders of means of discrete and continuous semigroups of operators, Studia Math. 187 (2008), 1-35.

[18] M. Lorente Domínguez and F. J. Martín-Reyes, The ergodic Hilbert transform for Cesàro bounded flows, Tohoku Math. J. (2) 46 (1994), 541-556.

[19] F. J. Martín-Reyes, New proofs of weighted inequalities for the one-sided HardyLittlewood maximal functions, Proc. Amer. Math. Soc. 117 (1993), 691-698.

[20] F. J. Martín-Reyes, P. Ortega Salvador and A. de la Torre, Weighted inequalities for one-sided maximal functions, Trans. Amer. Math. Soc. 319 (1990), 517-534.

[21] J. L. Rubio de Francia, Factorization theory and $A_{p}$ weights, Amer. J. Math. 106 (1984), 533-547.

[22] R. Sato, An Abel-maximal ergodic theorem for semigroups, Pacific J. Math. 51 (1974), 543-547.

[23] -, An ergodic maximal equality for nonsingular flows and applications, Acta Math. Hungar. 61 (1993), 51-58.

[24] E. Sawyer, Weighted inequalities for the one-sided Hardy-Littlewood maximal functions, Trans. Amer. Math. Soc. 297 (1986), 53-61.

H. Aimar, A. L. Bernardis

IMAL-CONICET

Güemes 3450

3000 Santa Fe, Argentina

E-mail: haimar@santafe-conicet.gov.ar

bernard@santafe-conicet.gov.ar
F. J. Martín-Reyes

Departamento de Análisis Matemático

Facultad de Ciencias

Universidad de Málaga

29071 Málaga, Spain

E-mail: martin_reyes@uma.es

Received May 7, 2009

Revised version May 7, 2010 